\title{
The heterogeneity of the left dorsal premotor cortex evidenced by multimodal connectivity-based parcellation and functional characterization.
}

Sarah Genon ${ }^{\mathrm{a}}$, Andrew Reid ${ }^{\mathrm{g}}$, Hai Li ${ }^{\mathrm{e}, \mathrm{f}}$, Lingzhong Fan ${ }^{\mathrm{e}, \mathrm{f}}$, Veronika I. Müller ${ }^{\mathrm{a}, \mathrm{c}}$, Edna C. Cieslik $^{\mathrm{a}, \mathrm{c}}$, Felix Hoffstaedter ${ }^{\mathrm{a}}$, Robert Langner ${ }^{\mathrm{a}, \mathrm{c}}$, Christian Grefkes ${ }^{\mathrm{b}, \mathrm{d}}$ Angela R. Laird ${ }^{\mathrm{h}}$, Peter T. Fox ${ }^{\mathrm{i}}$, Tianzi Jiang ${ }^{\mathrm{e}, \mathrm{f}}$, Katrin Amunts ${ }^{\mathrm{a}, \mathrm{j}}$ and Simon B. Eickhoff ${ }^{\mathrm{a}, \mathrm{c}}$.

anstitute of Neuroscience and Medicine (INM-1), Research Centre Jülich, Germany.

${ }^{\mathrm{b}}$ Institute of Neuroscience and Medicine (INM-3), Research Centre Jülich, Germany.

${ }^{\mathrm{c}}$ Institute of Systems Neuroscience, Medical Faculty, Heinrich-Heine University Düsseldorf, Germany.

${ }^{\mathrm{d}}$ Department of Neurology, Cologne University Hospital, Cologne, Germany.

${ }^{\mathrm{e}}$ Brainnetome Center, Institute of Automation, Chinese Academy of Sciences, Beijing 100190, China

${ }^{\mathrm{f}}$ National Laboratory of Pattern Recognition, Institute of Automation, Chinese Academy of Sciences, Beijing 100190, China

${ }^{\mathrm{g}}$ Department of Artificial Intelligence, Donders Centre for Cognition, Radboud University Nijmegen, The Netherlands.

${ }^{\text {h}}$ Neuroinformatics and Brain Connectivity Lab, Miami, Florida, USA.

${ }^{\mathrm{i}}$ Research Imaging Institute, University of Texas Health Science Center at San Antonio, TX, USA.

${ }^{\mathrm{j}} \mathrm{C}$. and O. Vogt Institute for Brain Research, Heinrich-Heine-University Düsseldorf, Düsseldorf, Germany.

Keywords: precentral gyrus, eye-field, meta-analytic connectivity modeling, probabilistic diffusion tractography, fMRI.

Corresponding author: Sarah Genon, Institut für Neurowissenschaften und Medizin (INM1), Forschungszentrum Jülich GmbH, D-52425 Jülich, Germany. E-mail: s.genon@fzjuelich.de. 
Genon et al.

\begin{abstract}
$\underline{\text { Abstract }}$
Despite the common conception of the dorsal premotor cortex (PMd) as a single brain region, its diverse connectivity profiles and behavioral heterogeneity argue for a differentiated organization of the PMd. A previous study revealed that the right PMd is characterized by a rostro-caudal and a ventro-dorsal distinction dividing it into five subregions: rostral, central, caudal, ventral and dorsal. The present study assessed whether a similar organization is present in the left hemisphere, by capitalizing on a multimodal data-driven approach combining connectivity-based parcellation (CBP) based on meta-analytic modeling, restingstate functional connectivity, and probabilistic diffusion tractography. The resulting PMd modules were then characterized based on multimodal functional connectivity and a quantitative analysis of associated behavioral functions. Analyzing the clusters consistent across all modalities revealed an organization of the left PMd that mirrored its right counterpart to a large degree. Again, caudal, central and rostral modules reflected a cognitivemotor gradient and a premotor eye-field was found in the ventral part of the left PMd. In addition, a distinct module linked to abstract cognitive functions was observed in the rostroventral left PMd across all CBP modalities, implying greater differentiation of higher cognitive functions for the left than the right PMd.
\end{abstract}


Genon et al.

\section{Introduction}

The dorsal premotor cortex (PMd) constitutes a transitional region between the primary motor and prefrontal cortex (Geyer et al. 2000), encompassing the dorsal portion of the precentral gyrus. While the PMd is usually referred to as a single brain region corresponding to Brodmann area 6 , its diverse connectivity profile and the heterogeneity of functions attributed to this region argue for functional and anatomical diversity within it (Boussaoud 2001; Picard and Strick 2001; Hoshi and Tanji 2007; Abe and Hanakawa 2009; Hanakawa 2011). Such diversity extends that of the lateral prefrontal cortex, which is organized along different axes (including the rostro-caudal; Badre 2008; Badre and D'Esposito 2009; Cieslik et al. 2013; Bahlmann et al. 2015)). Consistent with this view, a parcellation of the precentral gyrus based on probabilistic diffusion tractography (PDT-CBP) suggested that the dorsal part of the precentral gyrus, roughly corresponding to the functional PMd, can be further subdivided into a superior and an inferior portion (Schubotz et al. 2010). Thus, several lines of evidence converge to suggest that the PMd is not a uniform functional region, but rather a functional and/or an anatomical mosaic.

In a previous study, we investigated this issue in the right hemisphere (Genon et al. 2016) using connectivity-based parcellation (CBP) based on meta-analytic connectivity modeling (MACM-CBP; (Eickhoff et al. 2011)), resting-state functional connectivity (RSFC-CBP; (Yeo et al. 2011a)) and probabilistic diffusion tractography (PDT-CBP; (Behrens et al. 2003b)). Across modalities, we found a convergent subdivision of the right PMd into five modules organized along a rostro-caudal axis and a ventro-dorsal axis (Genon et al., 2016). Functional characterization of the defined subregions revealed that the rostral-caudal organization formed by the rostral, central and caudal subregions reflected a cognitive-motor gradient. The rostral subregion was associated with higher-order functions and connected to the prefrontal cortex, the central subregion showed a mixed pattern and connections to the parietal lobe, while the caudal subregion was related to motor functions and connected to the sensorimotor network. In turn, the dorsal subregion was related to both cognitive and motor aspects of sequencing and timing while the ventral subregion showed a functional profile implicating it as a "premotor eye field" (Genon et al. 2016).

It remains an open question to what extent the organization of the left hemisphere mirrors the organization of the right hemisphere. According to previous whole brain parcellations, the left 


\section{Genon et al.}

hemisphere showed a similar rostro-caudal organization to the right one, reflecting a general cognitive-motor gradient (Orban et al. 2015). There is also evidence that the frontal eye-fields should be similarly located in the ventral part of the PMd in both hemispheres (Amiez and Petrides 2009). However, the left hemisphere is known to be more functionally dominated by language functions (such as speech preparation and execution, e.g. (Kell et al. 2010)) than the right hemisphere and, in turn, less engaged in visuospatial processes (e.g. (De Schotten et al. 2011)) with such functional asymmetry including the PMd (Wager et al. 2004). Moreover, the left premotor cortex has been demonstrated to have a more pronounced engagement in bimanual coordination, motor learning (Hardwick et al., 2014) and mental calculations (Hanakawa 2011). Relatedly, eye movements and right hand movement are usually executed in unison (Kantak et al. 2012), possibly rendering the functional interaction within the left PMd more complex than in the right PMd. Based on these findings, we expected the functional organization of the left PMd to be different from the one previously demonstrated for the right PMd (Genon et al., 2016). In particular, we predicted that the functional segregation of eye-field functions and visuo-spatial integration would be less prominent in the left PMd, while language functions would be represented by a specific subregion in the left PMd. Supporting this possibility, Glasser at al. (2016) have recently proposed a "new" language subregion termed "area $55 \mathrm{~b}$ " in the inferior portion of both the left and right PMd. In our previous study, however, we did not find evidence of such a language subregion in the right PMd, highlighting the possibility that this subregion is specific to the left hemisphere. Importantly, previous functional studies have, albeit often indirectly, already suggested a different organization of the left and right PMd. For example, when examining activations of cognitive functions attributed to the PMd with a meta-analytic approach, Hanakawa (2011) concluded: "The right rostral premotor cortex was located 3-6 $\mathrm{mm}$ rostral to the left rostral premotor cortex consistently across different task sets." In the same line, previous wholebrain parcellations based on RSFC-CBP revealed slightly different patterns for the left and right PMd ((Yeo et al. 2011b; Laumann et al. 2015) see Supplementary Material). Nevertheless, such asymmetries may be related to peculiarities of functional imaging and resting-state modeling. Hence, a multi-modal approach is necessary to examine the presence of potentially subtle differences in the neurobiological organization of the left PMd.

We here used CBP (Eickhoff et al. 2015) in order to provide a robust picture of the organization of the left PMd. First, we investigated evidence for a topographical organization that is "modality independent" by employing three different connectivity modalities: MACM, 
Genon et al.

RSFC and PDT. MACM is a functional connectivity approach that capitalizes on the robust profile of co-activations of the brain voxels across a wide range of task-based fMRI and PET studies (Eickhoff et al. 2011). That is, MACM reveals how voxels interact, or are organized, to support a behavioral task (in an fMRI or a PET scanner). It thus provides clustering of voxels that is more "behaviorally meaningful" than other CBP approaches, but it comes with the limitation that the clustering might be driven by epiphenomena and peculiarities of taskbased neuroimaging experiments such as a dominance of right-hand responses. Furthermore, as MACM is based on a meta-analytic approach, it is subject to the possibility of a publication bias (Rothstein et al. 2006). In turn, RSFC provides a clustering that is driven by the behaviorally unconstrained functional connectivity pattern of the voxels (Foster et al. 2016). Notably, the effect of noise and non-neuronal signals in RSFC is still an open issue (e.g. (Birn 2012; Murphy et al. 2013)) and therefore spurious correlations may influence the clustering patterns of voxels based on RSFC. Importantly, as both MACM and RSFC are correlative approaches, functional connectivity profiles may be indirect (e.g. driven by a third region). PDT suffers less from these limitations as it estimates structural connectivity based on diffusion data (Behrens et al. 2003b). Therefore, PDT brings into account the structural constraints of brain networks. However, despite substantial developments in PDT methods over the last years, the pattern revealed by this approach is still sensitive to technical limitations such as gyral bias and questionable validity in estimating long-distance connection (Van Essen et al. 2013). In summary, RSFC-CBP reveals an unconstrained functional organization of the voxels, MACM-CBP reveals a behavior-driven organization of the voxels, and PDT-CBP reveals a structural organization of the voxels. Each connectivity approach has its own advantages and limitations, rendering them complementary and making their integration a powerful tool for characterizing brain organization. This issue is particularly relevant when addressing the organization of a brain region as complex as the left PMd (cfr. Figure S1).

By capitalizing on the complementarity of these connectivity modalities, the present study investigated subdivisions of the left PMd in a data-driven and multimodal manner. In addition, the current work capitalized on several recent developments in the field as compared to earlier work. First, MACM-CBP was performed on a higher number of available experiments in the Brainmap database. Second, RSFC-CBP and PDT-CBP were performed on a large, publicly available dataset of healthy adult subjects (enhanced NKI/Rockland sample). Finally, RSFC-CBP benefitted from recent developments in signal denoising 


\section{Genon et al.}

(Salimi-Khorshidi et al. 2014). Potentially even more important, however, is the fact that the current work represents the first multi-modal CBP that employs the same analysis pipeline across modalities, rendering the actual parcellation algorithms constant. As we found a good convergence across modalities for the right PMd, we expected a similar convergence for the left one given the aforementioned improvements. The next step of the current work was then to functionally characterize the identified modules forming the left PMd mosaic with multimodal functional connectivity and functional behavioral profiling (e.g.(Clos et al. 2013; Genon et al. 2016)). In summary, our approach aimed to provide the first robust modular map of the left PMd based on multi-modal CBP combined with a detailed characterization of the behavioral functions and connectivity of the identified modules. 
Genon et al.

\section{Methods}

\subsection{PMd Volume of interest (VOI)}

We used the same functional approach to the definition of a PMd VOI as previously described in detail for the right side (Genon et al., 2016). In short, we first merged PMd activation sites from multiple meta-analyses on functions attributed to the PMd such as action observation (Caspers et al. 2010), motor learning (Hardwick et al. 2013), movement perception (Grosbras et al. 2012), sustained attention (Langner and Eickhoff 2013), and working memory (Rottschy et al. 2012). The ensuing PMd VOI was symmetrized and exclusively masked with the cytoarchitectonic maps of primary sensorimotor areas (BA4a, $4 p 3 a, 3 b, 1,2)$ as provided by using the SPM Anatomy Toolbox (Eickhoff et al. 2005). This procedure ensured that the PMd VOI did not overlap with primary sensorimotor cortex at the caudal border. Rostrally, however, the PMd VOI was not restricted to Area 6 but rather covered the full extent of functionally defined PMd. This procedure resulted in a left PMd VOI of 4039 voxels.

\subsection{Connectivity computation}

We used MACM applied to the peaks of activation studies available in Brainmap database (https://www.brainmap.org/) as a robust measure of task-based functional connectivity while RSFC and PDT were computed respectively on the resting-state (RS) and diffusion-weighted imaging (DWI) data of 124 healthy adults ( 84 females; mean age $46.56 \pm 17.56$ ) from the open-access enhanced "Rockland" sample (http://fcon 1000.projects.nitrc.org/indi/enhanced).

\subsubsection{Meta-analytic connectivity modeling (MACM)}

MACM was used to identify the task-based functional connectivity profile of all left PMd VOI voxels as previously described (Clos et al. 2013; Genon et al. 2016). For each voxel within the left PMd all experiments that activated this voxel or its immediate vicinity were identified and whole-brain co-activation patterns computed using the BrainMap database. To this end, we first calculated the Euclidean distances between a given seed voxel and the individual foci of all experiments and then used a multi-filter approach including the closest 20 to 200 experiments in steps of two (i.e., retrieving the 20,22,24, 26,.., 200 experiments reporting activation closest to the seed voxel) to generate robust co-activation maps (Cieslik et al. 2013; Clos et al. 2013; Genon et al. 2016). The brain-wide co-activation profile for each seed voxel given each of the 91 filter sizes was then computed by a meta-analysis over the associated experiments. This meta-analysis was performed using the revised ALE algorithm 
Genon et al.

(Eickhoff et al. 2012). To take into account the complete brain-wide pattern of co-activation likelihood of each seed voxel, no height threshold was set. The brain-wide co-activation profiles for all left PMd VOI voxels were combined into a $N_{S} \times N_{B}$ connectivity matrix. $N_{S}$ is the number of seed voxels (4039) and $N_{B}$ the number of target voxels in the reference brain volume at $4 \times 4 \times 4 \mathrm{~mm}$ resolution (26459 grey-matter voxels). Altogether, 91 individual connectivity matrices were computed, each representing the connectivity of the seed voxels for a given filter size.

\subsubsection{Resting state functional connectivity (RSFC)}

Resting-state data (TR $1.4 \mathrm{~s}$ ) was cleaned for physiological and movement artifacts by applying FIX (FMRIB's ICA-based Xnoiseifier, version 1.061; (Salimi-Khorshidi et al. 2014)) implemented in FSL version 5.0.9 (http://fsl.fmrib.ox.ac.uk/fsl/fslwiki/FSL). FIX uses independent component analysis and automatically classifies noise components by using a large number of distinct spatial and temporal features via pattern classification. We utilized the training dataset provided with FIX and recommended settings for noise and movement regression (Griffanti et al. 2014). Further image processing was performed using SPM8 (Wellcome Trust Centre for Neuroimaging, London, http://www.fil.ion.ucl.ac.uk/spm/software/spm8). Following deletion of the first four scans preprocessing of the EPI images included affine registration to the mean EPI and normalization to MNI space using the unified segmentation approach before band-pass filtering for frequencies between 0.01-0.08 Hz. Linear (Pearson) correlations between the time series of each seed left PMd voxel and all other grey-matter voxels (at $4 \times 4 \times 4 \mathrm{~mm} 3$ resolution) were computed. The correlation coefficients were Fisher-Z transformed and defined a per-subject connectivity matrix in the same form as the (per filter) connectivity matrices obtained from the MACM computation.

Of note, one recent study (published after the time of our analyses) suggested that FIX should be optimally combined with global signal regression (Burgess et al. 2016). Therefore, we also performed Fix combined with linear global signal regression (GSR). However, addition of GSR did not impact on the subsequent RSFC-CBP parcellation as illustrated in Figure S5 in Supplementary Material. Furthermore, a high-quality dataset of RS fMRI data acquired in healthy young adults has been made available by the Human Connectome Project (HCP; http://www.humanconnectome.org/data/) after the time of our analysis. Therefore, we also performed RSFC-CBP on a sample from this dataset (324 young healthy adults, 164 females; mean age: $28.22 \pm 3.88$ ). This additional analysis yielded a similar parcellation pattern than the one obtained from the enhanced NKI sample as illustrated in Figure S6. 
Genon et al.

\subsubsection{Probabilistic Diffusion Tractography (PDT)}

Using FSL (http://www.fmrib.ox.ac.uk/fsl) the diffusion scans were first corrected for eddy current distortions and motion, followed by brain extraction based on the average b0 image and finally the estimation of a multi-fiber model using BEDPOSTX (which infers the existence of crossing fibers and estimates the contribution of each crossing fiber to the diffusion-weighted signal; cf. Behrens et al., 2007). In addition, linear and nonlinear transforms between diffusion and MNI-152 space were estimated using the FLIRT and FNIRT tools. Probabilistic Diffusion Tractography was then ran using Probtrackx (Behrens et al. 2003a; Behrens et al. 2007), generating 5000 streamline samples for every seed voxel based on the distributions of voxel-wise principal diffusion directions (using a curvature threshold of .2 and a step-length of $.5 \mathrm{~mm}$ ). Recording for every seed-voxel the streamlinecount at every voxel within the white-matter at a resolution of $3 \times 3 \times 3 \mathrm{~mm}$ then defined a persubject connectivity matrix in the same form as the (per subject) RSFC connectivity matrices and the (per filter) connectivity matrices obtained for MACM.

\section{3. k-means clustering}

For all the three connectivity modalities, the parcellation was performed by the same pipeline (for minor adjustments cf. Supplementary Material) using k-means clustering as implemented in the yael package (https://gforge.inria.fr/projects/yael). Given that we previously found a robust parcellation of the right PMd VOI into 5 clusters (Genon et al. 2016), we here investigated potential subdivisions obtained up to 7 clusters, i.e., the 2-cluster solution, 3cluster solution, and so on, up to the 7-cluster solution, That is, we a-priori assumed that meaningful organizations of the left PMd can be observed at low resolution and high resolution but not at very high resolution (i.e. not in subdivision into more than 7 subregions). For each parcellation, the best solutions from 500 replications with a randomly placed initial centroid were computed. Importantly, for each k, k-means clustering is performed at the individual level (i.e. filter level for MACM-CBP, and subject level for RSFC-CBP and PDTCBP) and the different parcellations are then combined into a single parcellation for each modality by computing the most frequent cluster assignment for each PMd VOI voxel across subjects/filter sizes.

\subsection{Cluster validity criteria}

Assuming that parcellations at a coarse scale (into 2-3 subregions) should represent the more stable primary patterns such as a rostro-caudal organization, we first considered these before 


\section{Genon et al.}

moving to finer parcellations with a particular focus on those close to the granularity of the solution obtained for the right side, i.e., $\mathrm{k}=4-6$. Importantly, however, at these finer scales, only some solutions should be expected to represent stable and hence supposedly meaningful subdivisions, necessitating an objective choice of the solution most supported by the data (Eickhoff et al., 2015). Here we employed four different cluster-validity metrics employed individually to all three modalities. In line with our parcellation of the right PMd VOI (Genon et al, 2016), we examined percentage of deviants and silhouette value. Of note, variation of information across filter sizes, which was investigated in our MACM-CBP of the right PMd VOI is a MACM-CBP specific metric, therefore it was not used in the current multimodal procedure. Rather, in the current multimodal CBP study, we additionally examined hierarchy index and change in inter/intra cluster distance (Clos et al. 2013). Thus, we examined four different criteria: a topological criterion (hierarchy index), a consistency criterion (percentage of deviants) and two cluster separation criteria (change in inter/intra cluster distance and silhouette value).

Hierarchy index: The topological criterion was the percentage of voxels not related to the dominant parent cluster compared to the previous $(\mathrm{k}-1)$ solution, i.e., the hierarchy-index (Kahnt et al., 2012). It corresponds to the percentage of lost voxels when only voxels consistent across the entire hierarchy are considered for the final clustering. For example, voxels assigned to cluster $\mathrm{X}$ in the 4 -cluster solution that were assigned to cluster $\mathrm{A}$ (at $\mathrm{k}=3$ ) would be excluded if the majority of cluster X voxels actually stemmed from cluster B (at $\mathrm{k}=3$ ). A large fraction of such voxels indicates a hierarchically unstable solution (Clos et al. 2013).

Percentage of deviants: The percentage of deviants or "misclassified voxels", i.e. the average percentage of voxels for each filter size/subject that were assigned to a different cluster compared to the most frequent (mode) assignment of these voxels across filter sizes/subjects, was used as a consistency criterion. A significant difference in percentage of deviants between a given cluster solution and the previous (k-1) one was tested using a two-sample ttest. Optimal solutions are those k parcellations where the percentage of deviants (presumably reflecting noise and local variance) is not significantly increased compared to the previous (k1) solution, while the subsequent $(\mathrm{k}+1)$ solution leads to a significantly higher percentage of deviants. 
Genon et al.

Change in inter/intra cluster distance: The inter/intra cluster ratio (Chang et al. 2012), that is, the ratio between the average distance of a voxel to its cluster centre and the average distance between the cluster centers, was used as cluster separation criterion. Since the higher the distance ratio, the better is the separation, a significant increased ratio compared to the previous k-1 solution would indicate a better separation of the obtained clusters. However, because of the monotonous increase usually observed with this ratio, we used the first derivative to evaluate the change in this ratio across solutions. A local optimum is reached when there is a significant increase in the change from the previous $\mathrm{k}-1$ to the current $\mathrm{k}$ solution while the subsequent $\mathrm{k}+1$ solution does not show a significantly larger increase.

Silhouette value. The silhouette value ranges from -1 to 1 and assesses, for each voxel, how similar the voxel is to others within the same cluster, versus, how similar this voxel is to voxels in other clusters regarding connectivity profile. A significant difference in the silhouette value between a given cluster solution and the previous one was tested with a twosample t-test. Cluster solutions were considered favorable if they show a significantly higher silhouette value, as compared to the previous (k-1) solution.

\subsection{Identification and characterization of multimodal modules within the left PMd}

To identify anatomical modules reflected by consistent subregion across the three modalities, we first identified the best solution for each of these and then performed a (spatial) minimumstatistic conjunction across the ensuing clusters (i.e. conjunctions of each cluster of each modality with all other clusters of all other modalities) to arrive at a final, multi-modal parcellation of the left PMd VOI. In order to only retain modules that reflect meaningful overlaps between the uni-modal clusters but not, e.g., stripe-like fringe area resulting from a slight displacement of the cluster border between modalities, we additionally employed a module-size criterion of 150 continuous voxels. That is, following the identification of the best solution per modality and the parcellation of the left PMd VOI based on each aspect of brain connectivity, we computed the intersection between all possible combinations of the ensuing MACM-CBP, RS-CBP and PDT-CBP clusters. We then retained only those intersections between clusters from different modalities, which yielded at least 150 continuous voxels, as our final, multi-modal modules. These modules were subsequently characterized by their multi-modal functional interaction patterns and the behavioral tasks engaging them in functional neuroimaging studies. 


\section{Genon et al.}

\subsubsection{Functional connectivity of multimodal modules}

Specific task-related functional connectivity (MACM)

For each obtained PMd module, an ALE meta-analysis was performed across all BrainMap experiments featuring at least one focus of activation within each of the derived modules using the same approach as described above. In contrast to the MACM underlying CBP, where ALE maps were not thresholded to retain the complete pattern of co-activation likelihoods, we here performed statistical inference to identify brain regions showing significant co-activations (correcting the cluster-level family-wise error rate (cFWE at $p<.05$, using a cluster-forming threshold at voxel level of $p<.001$ ).

To compare the brain-wide co-activation pattern between modules, we performed metaanalytic contrasts as previously described (Eickhoff et al., 2011, Genon et al., 2016). Finally, we identified the specific co-activation pattern for each module, that is, brain regions that were significantly more co-activated with a given module than with any of the other ones. This was achieved by performing a minimum-statistic conjunction across the results of the four contrasts between a given module and the remaining others.

\section{Specific resting-state functional connectivity}

To compute the significant RSFC of each multimodally defined module, the time series of a given module was represented (per subject) by the first eigenvariate of the resting-state time courses of all voxels attributed to this module. Linear (Pearson) correlations between the time series of each module and all other grey-matter voxels were computed and transformed into Fisher's z-scores. These Fisher's z-scores were entered into a flexible factorial model as implemented in SPM8 to test for consistency across subjects (main effect) as well as for significant differences between the modules (again correcting the cluster-level family-wise error rate (FWE at $p<.05$, using a cluster-forming threshold at voxel level of $p<.001$ ). As for MACM, we identified the specific connectivity pattern of each module by performing a minimum-statistic conjunction across the results of the four contrasts between a given module and the remaining others.

In order to identify the robust, modality-independent, (specific) functional connectivity pattern for each module (cf. Amft et al., 2014), we finally combined MACM and RSFC results for each module using a minimum-statistic conjunction. 


\section{Genon et al.}

\subsubsection{Behavioral functional characterization of multimodal modules}

As in previous work (e.g. (Clos et al. 2013; Genon et al. 2016)), functional characterization of the left PMd modules was performed using the "behavioral domain" (BD) and "paradigm class" (PC) meta-data within the BrainMap database (Laird et al. 2009) for those experiments that activate the respective module. BDs include the main categories cognition, action, perception, emotion, interoception, as well as their subcategories. In turn, PCs categorize the specific task employed. To robustly characterize the individual functional profile of each left PMd module, we combined quantitative "forward inference" and "reverse inference". In forward inference, a module's functional profile is assessed by identifying taxonomic labels for which the probability of finding activation in the respective module is significantly higher than finding activation for that label across the whole database by chance $(p<.05$ corrected for multiple comparisons using Bonferroni's method (Clos et al. 2013; Rottschy et al. 2013). That is, we tested whether the conditional probability of activation in a particular region given a particular label $[\mathrm{P}($ Activation|Task $)]$ was higher than the baseline probability of activating this particular region [P(Activation)]. In reverse inference, a module's functional profile was determined by identifying the most likely BDs and PCs given activation in a particular module, i.e., the likelihood $\mathrm{P}$ (Task|Activation). This likelihood can be derived from $\mathrm{P}$ (Activation|Task) as well as $\mathrm{P}($ Task) and $\mathrm{P}$ (Activation) using Bayes' rule. Significance (at $p$ $<.05$ corrected) was then assessed by means of a chi-squared test. In sum, forward inference assesses the probability of activation given a behavioral label, whereas reverse inference tests the probability of each behavioral label given an activation. For the sake of robustness, only behavioral labels that were significantly associated to the modules across both, forward and reverse inferences, were taken into account

\section{Results}

\subsection{Multimodal parcellation}

We first examined how each of the three CBP modalities divided the left PMd VOI at lower and higher scales. Then we investigated how the different cluster-solutions were supported by the data (using various cluster-stability criteria) within each CBP modality and combined the supported parcellations to finally identify multi-modal modules. Importantly, we compared the derived multimodal modules with the three subregions defined within the superior part of 
Genon et al.

Area 6 (Sigl et al. 2016) by independent mapping modality (cytoarchitecture) and with the different subregions found in by the recent multimodal mapping of the cerebral cortex (Glasser et al. 2016).

\subsubsection{Rostro-caudal organization of the left PMd at lower scales $(\mathrm{k}=2-3)$}

All three modalities first subdivided the left PMd VOI along the rostro-caudal axis at lower scales. At $\mathrm{k}=2$, all modalities differentiated a caudal part on the precentral gyrus from a rostral part. Of note, while the borders defining subregions based on functional data (i.e. MACM-CBP and RSFC-CBP) did not meet macroanatomical landmarks, the border between the rostral and the caudal subregions defined by PDT-CBP was found close to the precentral sulcus. At $\mathrm{k}=3$, all three modalities differentiated a caudal subregion mainly covering the precentral gyrus, a central region centered on the precentral sulcus and a rostral subregion anterior to the precentral sulcus (Figure 1).

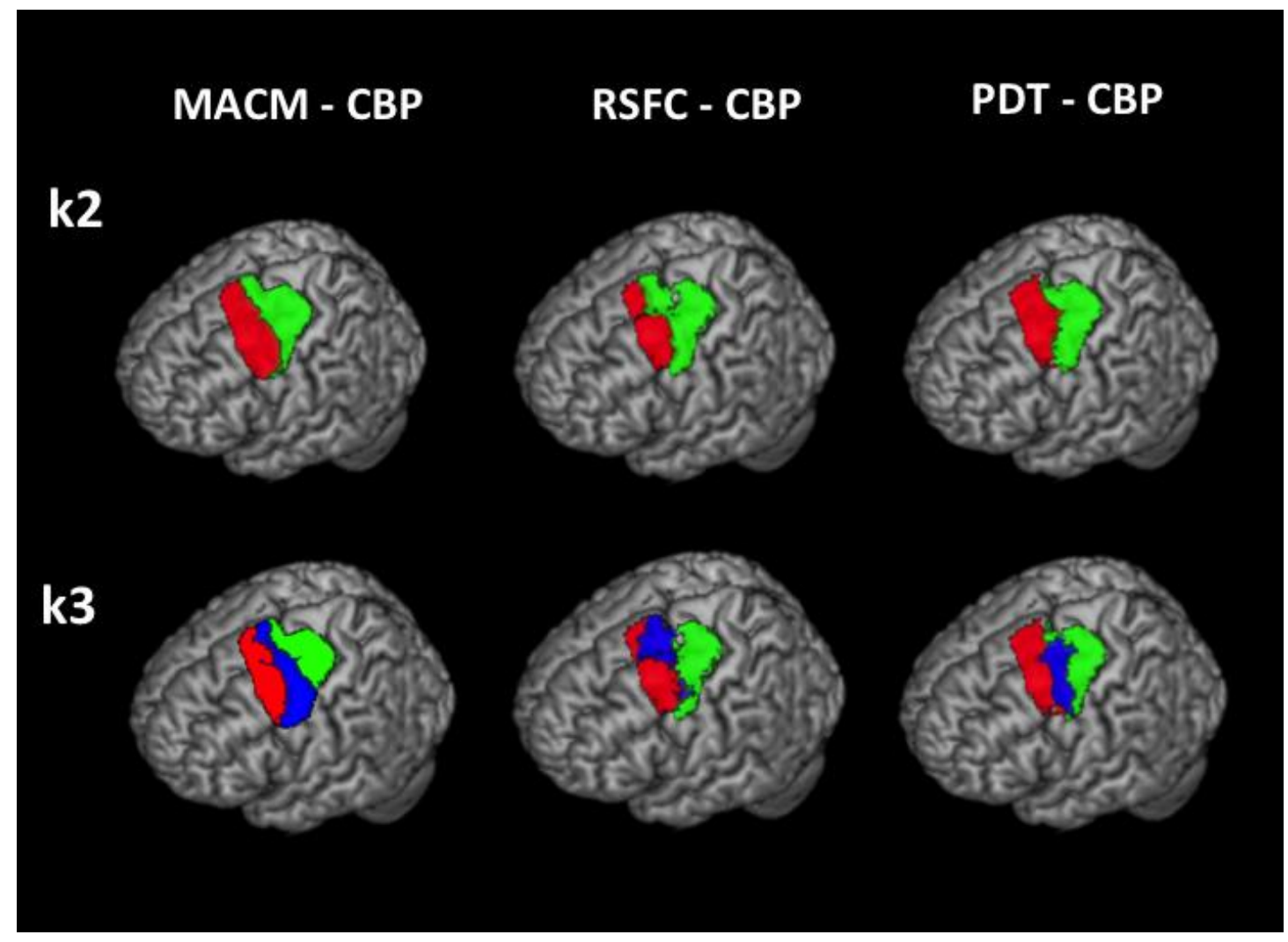

Figure 1: Rendering of the two subregions (k2, upper row) and three subregions (k3, lower row) of the left PMd yielded by the three CBP modalities. 
Genon et al.

As expected, these coarse parcellations tended to be more stable than finer parcellations in the subject-based modalities (i.e., RSFC-CBP and PDT-CBP, see Figure S4). In other words, the rostro-caudal organization into three subregions appeared as a more stable feature than further subdivisions within the left PMd VOI.

3.1.2. Ventro-dorsal organization of the left PMd at higher scales $(\mathrm{k}=4-6)$

Subsequent splitting of the PMd VOI highlighted differences as well as similarities between modalities (Figure 2).

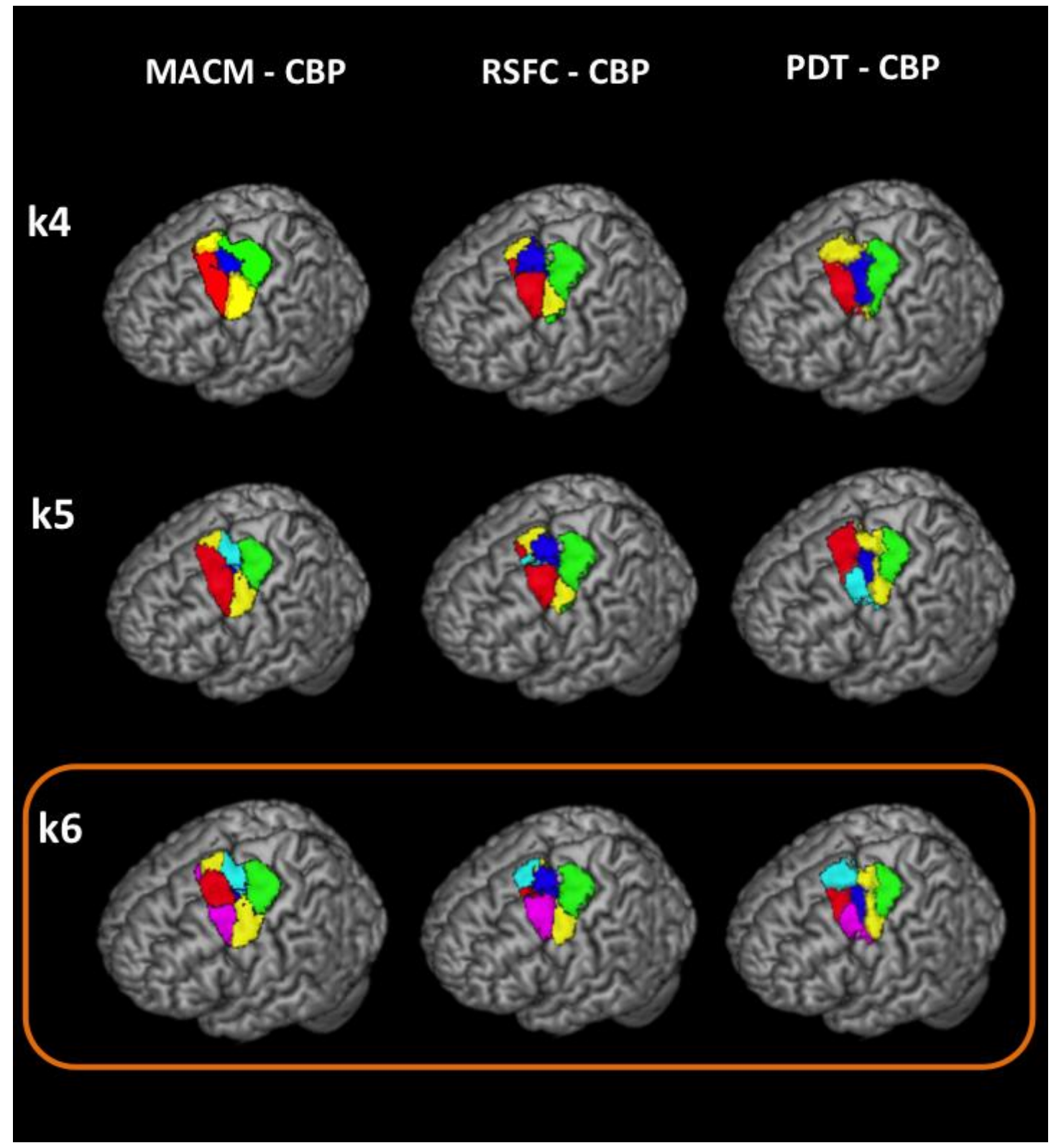




\section{Genon et al.}

Figure 2: Rendering of the subregions yielded by the three CBP modalities at high resolution $(\mathrm{k}>3)$. Please note that for MACM-CBP the deeper cluster (blue) in the superior frontal sulcus is not fully illustrated in the rendering $(\mathrm{k}=5$ and $\mathrm{k}=6)$. The orange frame denotes the three $6 \mathrm{k}$ parcellations that were combined for defining the multimodal modules.

All CBPs further subdivided the intermediate subregion (from $\mathrm{k}=3$ ) along the ventro-dorsal axis at $\mathrm{k}=4$. MACM and RSFC-CBPs both revealed that a "central" subregion, located at the intersection of the superior frontal and precentral gyri, can be distinguished from a more ventral and a more dorsal one as previously observed for the right PMd VOI (Genon et al., 2016). In contrast, PDT-CBP distinguished a superior part, roughly corresponding to the superior frontal sulcus portion of the left PMd VOI.

At $\mathrm{k}=5$, divergence between modalities increased. Both RSFC-CBP and PDT-CBP subdivided the rostral subregion into two modules along the ventro-dorsal axis disentangling a rostral module located in the superior sulcus either from a more rostroventral subregion (PDT-CBP) or from the cortex both rostro-ventral and rostro-dorsal to it (RSFC-CBP). In contrast, MACM-CBP subdivided the central cluster into a surface subregion and a deeper subregion, in the fundus of superior frontal sulcus, where it meets the precentral gyrus.

At $\mathrm{k}=6$, all CBPs further subdivided the superior rostral subregion along the ventro-dorsal axis. Both, RSFC-CBP and PDT-CBP isolated the extreme dorsal part. In turn, MACM-CBP distinguished the rostral module located on the superior frontal sulcus from the more rostroventral and rostro-dorsal subregions, in line with the subdivision revealed by RSFC-CBP at the previous clustering step $(\mathrm{k}=5)$.

Overall, for the 6 cluster solutions we identified across all three CBPs two rostral subregions: a rostral one located in the superior frontal sulcus (consistently with our previous CBPs of the right PMd VOI) and a rostro-ventral one, a central subregion located in the posterior superior frontal sulcus, where it meets the precentral gyrus, a ventral subregion and a caudal one adjacent to primary motor area.

\subsubsection{Selection of cluster solutions and cross-modal identification of modules within the left} $\underline{\mathrm{PMd}}$ 


\section{Genon et al.}

We next examined how the parcellations were supported by the data for the higher scales $(\mathrm{k}=$ 4-6) independently for each CBP modality (MACM, RSFC and PDT; see Supplementary Material for illustration and a detailed description of the results of the cluster stability assessment).

MACM-CBP: whereas the percentage of deviants and the silhouette value were not informative for choosing a cluster solution at higher scale (see Supplementary Material), the two other criteria promoted the $\mathrm{k}=6$ parcellation over other.

RSFC-CBP: whereas the intra/inter distance ratio, the percentage of deviants and the silhouette value were not informative for choosing a cluster solution at higher scale, the hierarchy index promoted the $\mathrm{k}=4$ and the $\mathrm{k}=6$ parcellations over the $5 \mathrm{k}$ parcellation.

PDT-CBP: whereas the percentage of the intra/inter distance ratio was not informative for choosing a cluster solution at higher scale, the silhouette value promoted the $\mathrm{k}=5$ parcellationat higher scale. Nevertheless, the two other criteria promoted the $\mathrm{k}=6$ parcellation over other $\mathrm{k}$ parcellations.

Thus, both within MACM-CBP and PDT-CBP, $\mathrm{k}=6$ can be regarded as the most stable solution. Of note the $6 \mathrm{k}$ was also supported by examination of the percentage of voxels not with parents for RSFC-CBP (despite the $4 \mathrm{k}$ was also supported). Thus, the $6 \mathrm{k}$ appeared as a data-supported cluster solution within each modality. Importantly, MACM-CBP and PDTCBP modalities that similarly promoted the $6 \mathrm{k}$ are based on different data sets. Therefore, in the sake of cross-modality validity, we focused on the $\mathrm{k}=6$ solution to identify multimodal modules.

To identify modules consistent across the three topological organizations and infer the multimodal organization of the left PMd, we performed conjunction analyses between all possible combination of MACM, RSFC and PDT clusters. This procedure revealed 5 modules (Figure 3): a caudal one (green, 400 voxels), a central one (blue, 333 voxels), a rostral one (red, 241 voxels), a ventral one (yellow, 158 voxels) and a rostro-ventral one (pink, 260 voxels). The volumes are available through ANIMA (http://anima.fz-juelich.de/, (Reid et al. 2016)). These five modules were consistent with the corresponding five subregions revealed by each CBP modality independently, while the surface subregion revealed by MACM-CBP alone and the dorsal subregion revealed by RSFC-CBP and PDT-CBP were not evidenced by the conjunction across CBP modalities. 


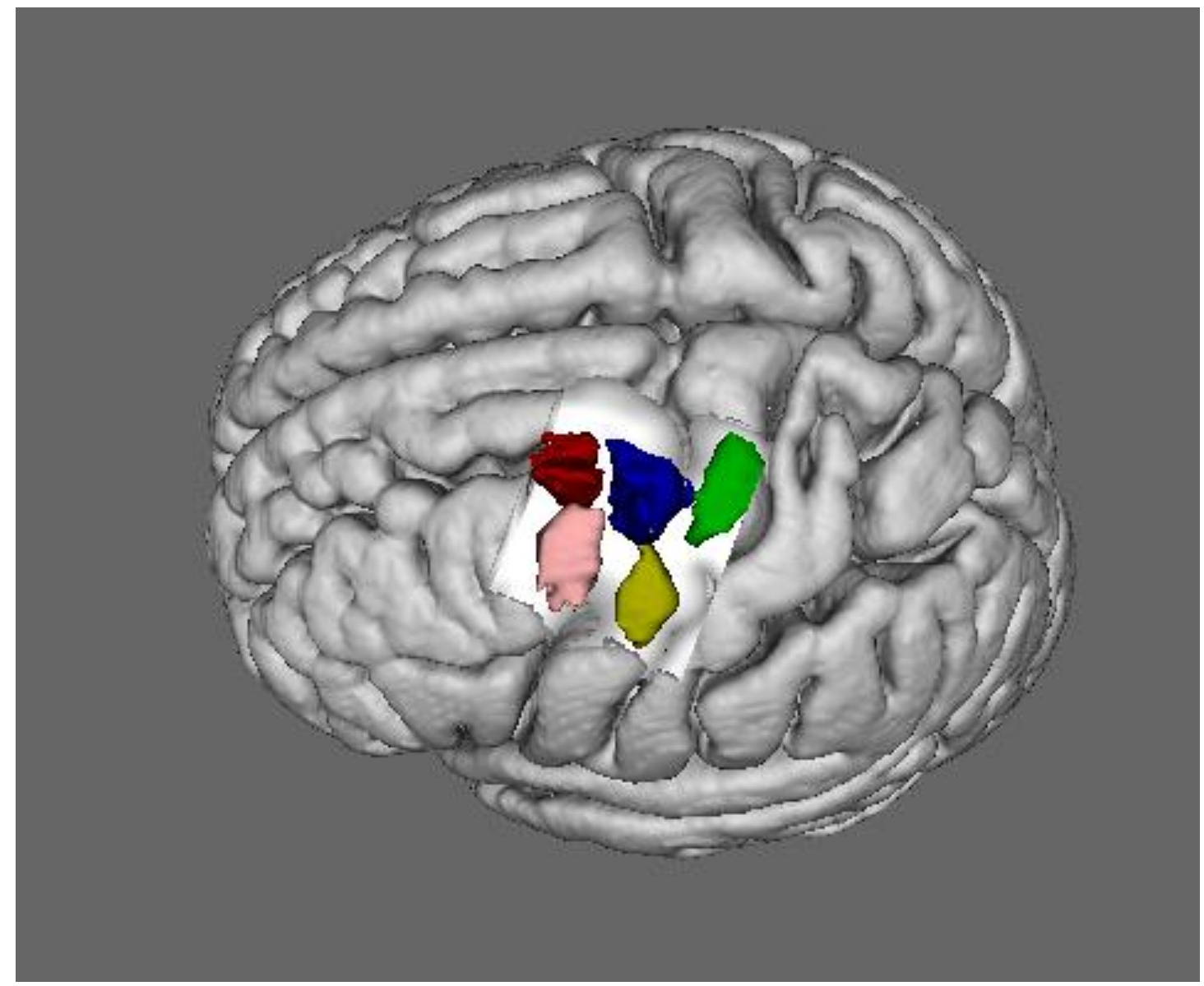

Figure 3. The five left PMd modules identified by multimodal CBP: red, rostral subregion; pink, rostro-ventral subregion; blue, central subregion; green, caudal subregion; yellow, ventral subregion. The five volumes are available in MNI space in the ANIMA database (http://anima.fz-juelich.de/).

\subsubsection{Comparison with cytoarchitecture-based mapping and multimodal cortex parcellation}

Recent cytoarchitecture-based mapping of the superior part of Area 6 has revealed three distinct areas: a caudal one (6d1), a deep anterior one (6d3) and a more dorsal anterior one (6d2) (Sigl et al. 2016). The comparison of our two multimodal modules located in the superior part of Area 6, i.e., the caudal and the central one, with the caudal and deep anterior anatomical areas showed that our caudal and central modules are consistent in their spatial pattern with these anatomical areas (this comparison is illustrated in Figure S7 in the Supplementary Material). Thus, the comparison of our resulting modules with ex-vivo microstructure-based mapping suggests that our conjunction of overlapping clusters across CBP modalities retains topographically valid patterns. 


\section{Genon et al.}

Recently, Glasser et al. (2016) proposed a surface-based multimodal parcellation of the cerebral cortex. In order to reliably relate our multimodal left PMd volumes to the multimodal surface parcels of Glasser et al., we performed a two-fold comparison (see Figure S8). We transposed the surface parcellation map of Glasser et al. to volume in MNI space and displayed our volumes on the volume map (Figure S8 A), but we also mapped our volumes to surface with the Connectome workbench (http://www.humanconnectome.org/software/connectome-workbench.html) and displayed the yielded surface modules on the surface parcellation map of Glasser et al. (Figure S8 B). Both comparisons showed that our left PMd caudal module mirrors a caudal PMd subregion identified by Glasser et al. In contrast, both comparisons showed that our rostral and central modules have not been distinguished in Glasser et al.'s parcellation as they are both part of a single parcel located within the posterior part of the superior frontal sulcus in this map. Both comparisons similarly suggest that our rostro-ventral module belongs to a larger, rather prefrontal, parcel. In turn, volume definition of area 55b from Glasser et al. suggests that this region corresponds to our ventral module while surface mapping of our ventral module shows that it overlaps with both Area 55b and the area superior to this latter.

\subsection{Functional characterization of left PMd modules}

\subsubsection{Multimodal functional connectivity}

The specific cross-modal functional connectivity patterns of each module are illustrated in Figure 4 and detailed in Table 1. For reader's information, the task-based functional connectivity (MACM) and resting-state functional connectivity (RSFC) are illustrated separately in Figure S9 and Figure S10, respectively, in Supplementary Material. Furthermore, the corresponding maps are available in ANIMA (http://anima.fz-juelich.de/). 
Genon et al.

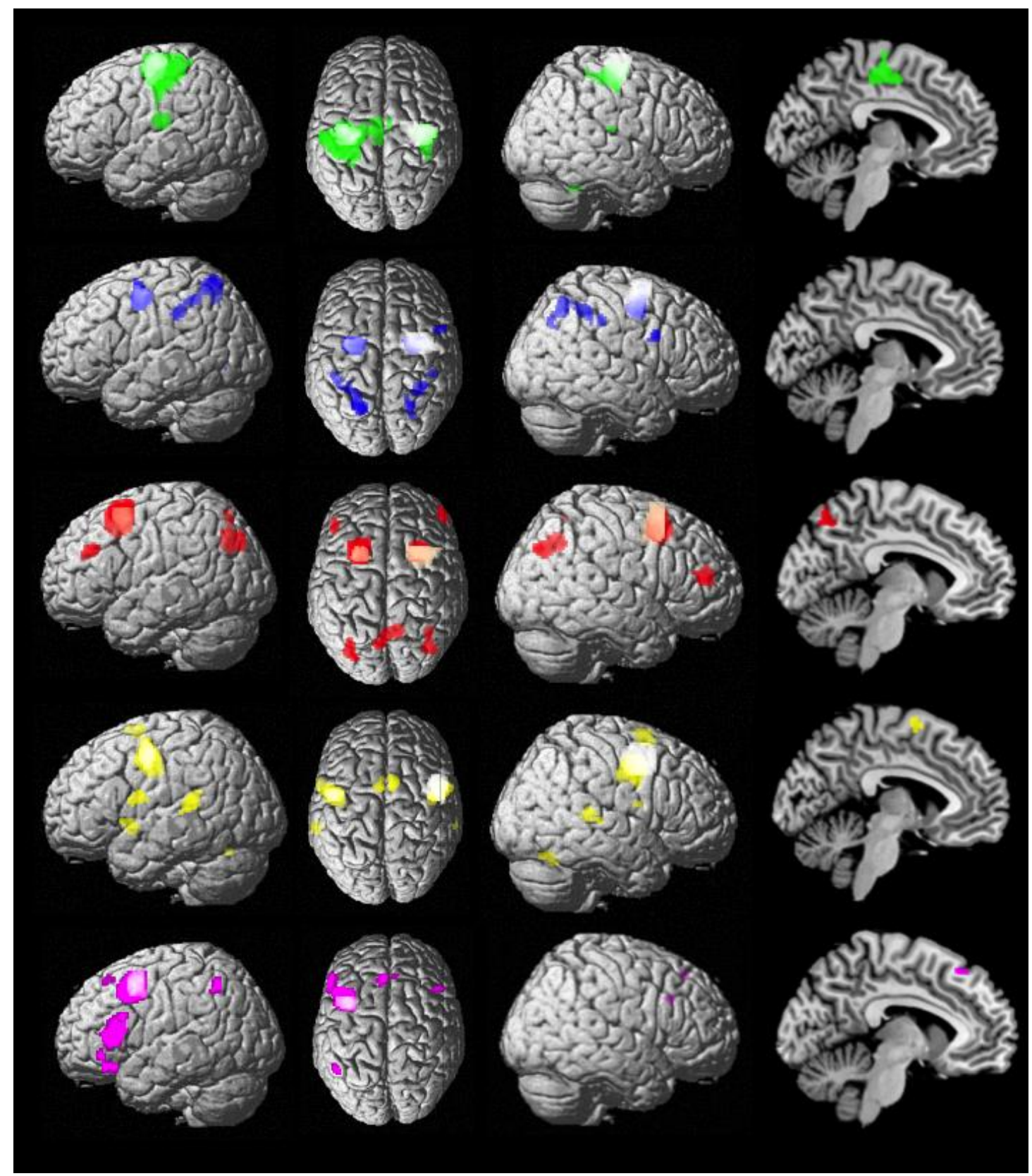

Figure 4. Specific functional connectivity of the five left PMd modules: green, caudal; blue, central; red, rostral; yellow, ventral; violet, rostro-ventral. The left multimodal PMd modules and the right PMd clusters (previously identified in Genon et al., 2016) are illustrated as white blobs. 
Genon et al.

Table 1. Specific functional connectivity of the five multimodal modules across tasks

(MACM) and rest (RSFC).

\begin{tabular}{|c|c|c|c|c|c|c|}
\hline Region & & $\begin{array}{c}\text { Overlap with } \\
\text { cytoarchitectonic area }\end{array}$ & $\mathrm{x}$ & $\mathrm{y}$ & $\mathrm{z}$ & $\begin{array}{l}\text { Cluster } \\
\text { size }\end{array}$ \\
\hline \multicolumn{7}{|l|}{$\begin{array}{l}\text { Specific to } \\
\text { caudal PMd }\end{array}$} \\
\hline \multirow[t]{3}{*}{ Primary sensorimotor cortex } & $\mathrm{L}$ & Area $3 b^{d} / 4 p^{c}$ & -31 & -22 & 58 & 2502 \\
\hline & $\mathrm{R}$ & Area $3 b^{d} / 4 p^{c}$ & 38 & -21 & 59 & 346 \\
\hline & & Area $2^{b} / 3 b^{e}$ & 40 & -39 & 60 & 12 \\
\hline \multirow[t]{2}{*}{ SMA } & $\mathrm{R}$ & NA & 5 & -7 & 51 & 24 \\
\hline & $\mathrm{L}$ & NA & -4 & -16 & 68 & \\
\hline Cerebellum & $\mathrm{R}$ & Lobule V/VI ${ }^{\mathrm{g}}$ & 19 & -52 & -21 & 419 \\
\hline Rolandic operculum & $\mathrm{L}$ & Area $\mathrm{OP} 1 / \mathrm{OP} 3 / \mathrm{OP} 4^{\mathrm{e}}$ & -48 & -22 & 19 & 216 \\
\hline \multicolumn{7}{|l|}{$\begin{array}{l}\text { Specific to } \\
\text { central PMd }\end{array}$} \\
\hline \multirow[t]{3}{*}{ Superior parietal lobule/IPS } & $\mathrm{L}$ & Area $7 \mathrm{~A}^{\mathrm{f}} /$ Area $7 \mathrm{PC}^{\mathrm{f}} / \mathrm{PFt} / 5 \mathrm{~L}^{\mathrm{f}}$ & -27 & -53 & 52 & 404 \\
\hline & $\mathrm{R}$ & Area $h l P 3^{\mathrm{i}}$ & 24 & -62 & 48 & 127 \\
\hline & & Area hlP $3^{\mathrm{i}} /$ Area $2^{\mathrm{b}}$ & 33 & -44 & 47 & 121 \\
\hline Supramarginal gyrus & $\mathrm{R}$ & Area $\mathrm{PFt}^{\mathrm{h}}$ & 45 & -32 & 40 & 24 \\
\hline Inferior Frontal gyrus & $\mathrm{R}$ & Area $44^{\mathrm{a}}$ & 51 & 9 & 29 & 56 \\
\hline $\begin{array}{l}\text { Right homotope of left } \\
\text { central PMd }\end{array}$ & $\mathrm{R}$ & NA & 28 & -4 & 53 & 339 \\
\hline \multicolumn{7}{|l|}{ rostral PMd } \\
\hline $\begin{array}{l}\text { Right homotope of left } \\
\text { rostral PMd }\end{array}$ & $\mathrm{R}$ & NA & 27 & 12 & 52 & 441 \\
\hline \multirow[t]{2}{*}{ Inferior parietal lobule } & $\mathrm{R}$ & Area $\mathrm{PGp}^{\mathrm{h}} / \mathrm{NA}$ & 37 & -69 & 37 & 129 \\
\hline & $\mathrm{L}$ & NA & -32 & -78 & 35 & 115 \\
\hline Superior parietal lobule/ & $\mathrm{L}$ & Area $7 \mathrm{~A}^{\mathrm{f}} / 7 \mathrm{P}^{\mathrm{f}}$ & -6 & -68 & 51 & 117 \\
\hline Precuneus & $\mathrm{R}$ & NA & 8 & -60 & 51 & 100 \\
\hline \multirow[t]{2}{*}{ Middle Frontal } & $\mathrm{R}$ & NA & 47 & 43 & 14 & 50 \\
\hline & $\mathrm{L}$ & NA & -45 & 36 & 27 & 27 \\
\hline
\end{tabular}


Genon et al.

\begin{tabular}{|c|c|c|c|c|c|c|}
\hline $\begin{array}{l}\text { Specific to } \\
\text { ventral PMd }\end{array}$ & & & & & & \\
\hline Superior temporal/higher & $\mathrm{L}$ & Area TE $3^{\mathrm{i}}$ & -59 & -37 & 14 & 144 \\
\hline & $\mathrm{R}$ & Area TE $3^{\mathrm{i}}$ & 59 & -33 & 8 & 55 \\
\hline Temporal pole & $\mathrm{L}$ & Area TE $3^{\mathrm{i}}$ & -53 & 8 & -3 & 44 \\
\hline $\begin{array}{l}\text { Right homotope of left } \\
\text { ventral PMd }\end{array}$ & $\mathrm{R}$ & NA & 50 & -3 & 42 & 427 \\
\hline Pre-SMA & $\mathrm{R}$ & NA & 7 & 6 & 65 & 126 \\
\hline & $\mathrm{L}$ & NA & -5 & 2 & 64 & 73 \\
\hline Inferior frontal & $\mathrm{L}$ & Area $44^{\mathrm{a}}$ & -58 & 2 & 17 & 73 \\
\hline Cerebellum & $\mathrm{R}$ & Lobule VI ${ }^{\mathrm{g}}$ & 29 & -64 & -22 & 58 \\
\hline Specific to & & & & & & \\
\hline Rostro-ventral PMd & & & & & & \\
\hline Inferior frontal cortex & $\mathrm{L}$ & Area $45^{\mathrm{a}}$ & -51 & 24 & 13 & 316 \\
\hline & $\mathrm{R}$ & NA & 46 & 21 & 33 & 20 \\
\hline Lateral orbitofrontal cortex & $\mathrm{L}$ & NA & -43 & 32 & -12 & 79 \\
\hline $\begin{array}{l}\text { Dorsomedial prefrontal } \\
\text { cortex }\end{array}$ & $\mathrm{L}$ & NA & -5 & 30 & 52 & 49 \\
\hline Inferior parietal lobule/IPS & $\mathrm{L}$ & NA & -48 & -55 & 48 & 41 \\
\hline
\end{tabular}

Note. ${ }^{a}$ Amunts et al., 1999; ${ }^{b}$ Grefkes et al., 2001; ${ }^{\circ}$ Geyer et al., 1996; ${ }^{\mathrm{d}}$ Geyer et al., 1999; ${ }^{\mathrm{e}}$ Eickhoff et al., 2006; ${ }^{\mathrm{f}}$ Scheperjans et al., 2008; ${ }^{\mathrm{g}}$ Diedrichsen et al., 2009; ${ }^{\mathrm{h}}$ Caspers et al., 2006; ${ }^{\mathrm{i}}$ Morosan et al., 2005; NA, not assigned to any known probability map/not applicable; PMd, dorsal Premotor Cortex; IPS, intraparietal sulcus; SMA, supplementary motor area.

The caudal module was specifically coupled with bilateral primary sensorimotor areas, secondary somatosensory cortex and cerebellum. The central module compared to all other modules was stronger connected with bilateral superior parietal cortex/IPS, but it also with the right supramarginal and inferior frontal gyri. In contrast, the rostral module was specifically coupled with bilateral middle frontal cortex, precuneus and inferior parietal cortex. In turn, the ventral module was functionally stronger connected with bilateral superior temporal cortex and pre-SMA, as well as left Broca's Area, left temporal pole and right cerebellum. 


\section{Genon et al.}

Finally, the rostro-ventral cortex was specifically connected with bilateral inferior frontal, left lateral orbitofrontal, dorsomedial prefrontal and inferior parietal cortices.

\subsubsection{Behavioral association: Behavioral domains and paradigm classes}

Functional characterization across behavioral domains and paradigm classes of the BrainMap database are summarized in Figure 5. This functional characterization revealed that the caudal module was mainly associated with motor-related functions while the rostral module was mainly associated with executive cognitive- and visuospatial- related functions. The central subregion, in turn, showed a mixed behavioral profile including motor and visuospatial functions and paradigms, but also related to working memory and attention. In contrast, the ventral module was mainly associated with speech and also eyes-related functions. Finally, the rostro-ventral module was associated with functions and paradigms requiring abstraction abilities, namely long term explicit memory, scene imagination and deception. 
Genon et al.

\section{Caudal Central Rostral Ventral Rostro-}

Behavioral Domains

Percep5on Somestesis

Ac5 on execu5 on

Motor learning

$A=$ en 5 on

Spa5al cogni5 on

Ac5 on imagina5on

Mo5on vision

Working memory

Reasoning

Speech cogni5 on

Speech execu5on

Music cogni5 on

Explicit memory

Paradigm Classes

TMS

Flexion/extension

Grasping

Poin5ng

Tac5le monitor discrimina5 on

Finger tapping

Sequenc recall/learning

Mental rota5 on

Imagined object/scene

Visuospa5al a $=$ en 5 on

Visual Object iden 5 fica5 on

Saccades

An5-saccades

Reading (overt)

Recita5on/repe55on

Tower of London

$\mathrm{N}$-back

Coun5ng/calcula5on

Reasoning/problem solving

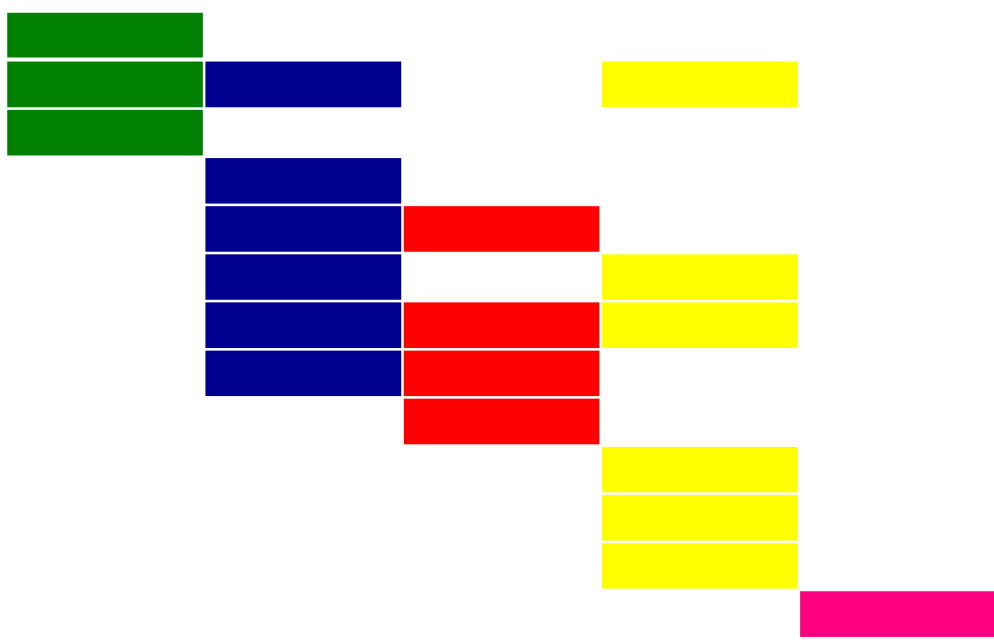

Decep5on
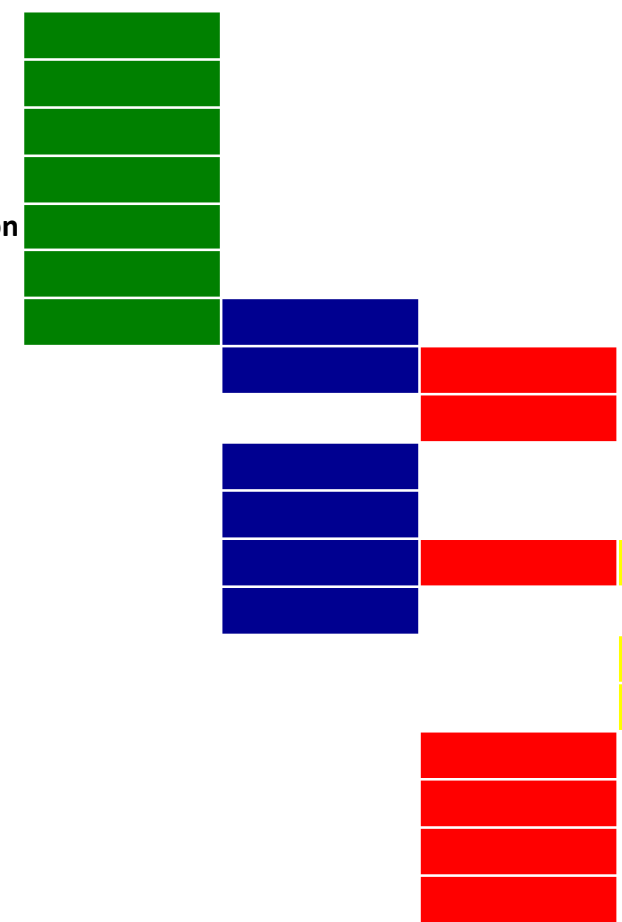

Figure 5. Functional decoding across behavioral domains and paradigm classes of the

BrainMap database of the five multimodal modules of the left PMd. 
Genon et al.

\section{Discussion}

In this study, we addressed the heterogeneity of the left PMd using a multimodal connectivity-based parcellation (CBP) approach combining meta-analytic connectivity modeling (MACM), resting-state functional connectivity (RSFC), and probabilistic diffusion tractography (PDT). At the coarser level - that is, when dividing the PMd into two and then three subregions based on the respective connectivity profiles of its individual voxels - all three modalities congruently revealed that the left PMd is organized along the rostro-caudal axis. At finer levels, when searching for further subdivisions, some divergence, as well as similarities, were observed across modalities. By crossing modalities and identifying voxels that are kept clustered together across parcellation modalities, we showed that five modules could be robustly distinguished within the left PMd. The five PMd subregions identified by the conjunction analyses corresponded to independent parcels within each CBP modality, suggesting that none of them could be considered as a conjunction artifact across modalities. In other words, all the five subregions identified by the conjunction analysis showed correspondence with the topographical organization revealed independently by the different modalities, thus truly reflecting convergence on the organization of the left PMd. These five subregions included a caudal module, a central module, a rostral module, a ventral module, and a rostro-ventral module.

\subsection{Topographical similarities and differences between right and left PMd}

The main modules identified in the right and left PMd multimodal CBP are illustrated in Figure 6.

[Figure 6]

Figure 6. Schematized PMd topography revealed by right (Genon et al., 2016) and left PMd parcellations.

The convergent organization across modalities found in the left PMd showed correspondence with the topographical organization previously highlighted in the right hemisphere, in which corresponding caudal, central, rostral and ventral subregions were also identified by different CBP modalities. In line with the rostro-caudal clusters identified in the right PMd, the left rostral subregion was mainly anterior to the precentral sulcus, the central subregion was found 


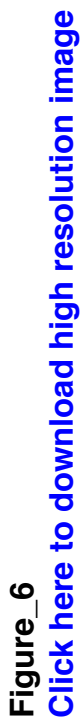
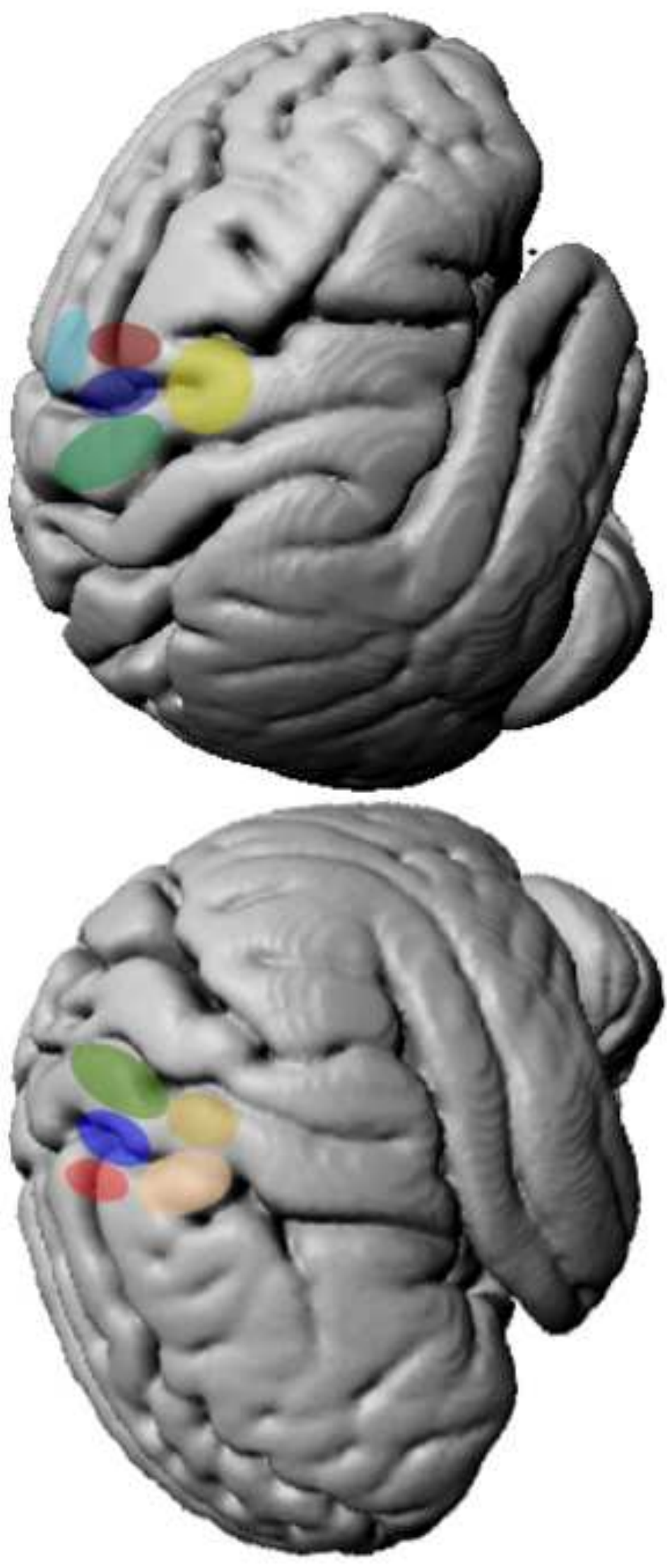


\section{Genon et al.}

at the level of the precentral sulcus and the caudal subregion was located on the precentral gyrus. In addition, in line with the ventral right PMd subregion, the ventral left PMd subregion was located on the ventral part of the middle portion of the precentral gyrus, bordering the ventral PMd. Nevertheless, while in our previous study of the PMd in the right hemisphere, different CBP modalities all isolated a dorsal subregion located on the superior frontal sulcus adjacent to pre-SMA, such a dorsal region was hardly distinguished from other subregions in the left PMd. In particular, MACM-CBP maintained the extreme dorsal voxels functionally linked to, on the one hand, the rostro-ventral subregions, and on the other hand, the ventral subregion. These findings suggest that the voxels on the dorsal part of the superior frontal gyrus of the PMd may not form a coherent functional module in the left PMd. In turn, all our CBP modalities and the subsequent conjunction analysis in the left PMd isolated a rostro-ventral module located anteriorly to the precentral sulcus on the ventral part of the middle frontal gyrus that was not evidenced in the right PMd.

\subsection{Evidence for hemispheric asymmetry of the right and left PMd}

In order to further examine whether asymmetry of the right and left PMd were truly supported by the data and could not be driven by methodological artifacts, we performed additional parcellation whose results are reported and described in Supplementary Material. First, to ensure that the method differences did not account for the differences in organization found in the two independent parcellations of the left and right PMd, CBP of the right PMd was performed with exactly the same methods and dataset as used for the left PMd. The 5-cluster parcellations found in the right PMd did not mirror the 5-cluster parcellation found in the left PMd (neither in Genon et al.'s original study nor with the improved method in Supplemental Material). In particular, whereas the ventral part of the left PMd was already subdivided into two subregions with a substantial rostro-ventral subregion in the left PMd, this rostro-ventral subregion was not evidenced in any of the right PMd parcellations (see Supplementary discussion III.2 and Figure S11). Second, we investigated whether a rostro-ventral subregion could be evidenced in the right PMd when further splitting this region into six clusters. However, this further subdivivsion revealed an inconsistent pattern across modalities (see Supplementary discussion III.2 and Figure S12), thus not supporting the hypothesis of a robust rostro-caudal subdivision in the inferior part of the right PMd. Third, in order to further investigate the hemispheric specificity hypothesis of the right dorsal PMd subregion and the left rostro-ventral PMd subregion, we examined how the PMd subregions could be clustered together across both hemispheres according to their behavior-related functional similarity. 
Genon et al.

That is, we performed MACM-CBP on a bilateral PMd VOIs. The main findings (which are described and illustrated in Supplementary Material, Figure S13) confirmed that the right dorsal PMd subregion and the left rostro-ventral PMd subregion did not show respective homotopic subregions in the opposite hemisphere. Thus, altogether, these additional findings support the hypothesis of different topographical organizations in the right and left PMd. The functional characterization of each left PMd module and their (lack of) correspondence to the right PMd subregions are further discussed below.

\subsection{A Rostro-caudal organization mapping cognitive-motor gradient in the left PMd}

At lower resolution $(\mathrm{k}=2-3)$, all CBP modalities highlighted a rostro-caudal organization, which could also be found at higher resolution $(\mathrm{k}=5-6)$ as three rostro-caudally organized modules on the superior part of the PMd: rostral, central and caudal. The ensuing functional characterization indicated a gradient from cognitive to motor functions with the rostral part being mainly connected to inferior parietal and lateral prefrontal cortex and associated with higher cognitive and visuospatial functions; the central part being associated with the topdown control network (Vossel et al. 2014) and being engaged in a wide range of behavioral functions from action execution to working memory; and the caudal part being functionally integrated in a sensorimotor network and associated with sensorimotor functions. The identified modules and their functional characterization bear similarities with the subregions described in non-human primates. The rostral module indeed mirrors area F7 (Matelli et al. 1985, 1991), a region predominantly receiving prefrontal inputs (Boussaoud et al. 1995; Rizzolatti and Luppino 2001) and supporting cognitive functions such as spatial attention or memory (Boussaoud 2001; Lebedev and Wise 2001; Nakayama et al. 2016). Similarly, the central module likely corresponds to F2vr (for a review see Abe and Hanakawa 2009), a subregion receiving inputs from dorsolateral prefrontal cortex (DLPFC) and medial IPS (Luppino et al. 2003), and assumed to support the integration of visuospatial and somatosensory/motor functions to complete a motor plan (for a review see Abe and Hanakawa 2009). The caudal module mirrors F2 itself, a subregion connected to M1 and the spinal cord (for reviews see Geyer et al. 2000; Abe and Hanakawa 2009). Furthermore, our multimodal characterization along the rostro-caudal axis is consistent with previous parcellations of the frontal lobe showing a cognitive-motor gradient mapping a rostro-caudal organization of the frontal cortex (e.g. (Koechlin and Summerfield 2007; Bellec et al. 2010; Yeo et al. 2011c; Orban et al. 2015; Glasser et al. 2016) as well as with our previous 
Genon et al.

parcellation of the right PMd (Genon et al. 2016), thus confirming that the rostro-caudal organization of the PMd is a primary principle of organization within the frontal cortex.

\subsection{The left premotor eye-field $55 \mathrm{~b}$}

On the ventral part of the middle frontal gyrus, our multimodal parcellation of the left PMd identified a ventral module that shows correspondence with the ventral subregion previously observed in the right PMd. Functional characterization of the left PMd ventral module showed a behavioral pattern dominated by visual and language-related functions. Accordingly, functional connectivity across MACM and RSFC showed that the ventral left PMd is functionally coupled with Broca's area and TE areas assigned to the ventral processing stream supporting object recognition (Gross 1994). When examining the functional profile of the left PMd ventral module and its spatial correspondence with the right PMd premotor eye-field, it appeared that the left PMd ventral module conceptually overlapped with the left premotor eye-field. In support of this view, a recent meta-analysis has identified a premotor eye-field located in our left PMd ventral module (Cieslik et al. 2016) as illustrated in Figure S14 (Supplementary Material). Nevertheless, the left premotor ventral module appeared additionally related to language functions and overlaps with Area 55b, a language-related area recently suggested by Glasser et al. (2016). However, behavioral functional characterization of the volume definition of Area 55b across the BrainMap database (reported in Supplementary Material) revealed that this subregion is also associated with simple eye movements and shows a similar profile to our ventral cluster (see Figure S15). Thus, the current evidence converges to suggest that the ventral subregion of the left PMd consists jointly of premotor eye-field and Area 55b. Such a mixed behavioral profile could reflect the integration/coordination of visual identification (visual screening) processes with word production (speech execution) processes in the ventral PMd, although future studies are needed to empirically confirm this hypothesis.

\subsection{Abstraction in the rostro-ventral left PMd}

At finer levels of parcellation, all CBP modalities isolated a rostro-ventral module located anteriorly to the precentral sulcus on the ventral part of the middle frontal gyrus, which was accordingly delineated by our conjunction analysis. Importantly, previous whole-brain parcellations have already suggested a finer subdivision of the inferior part of the PMd in the left hemisphere when compared to right hemisphere ((Yeo et al. 2011b; Laumann et al. 2015), 


\section{Genon et al.}

see Supplementary Material). The subsequent characterization of our delineated rostro-ventral module highlighted a conspicuous functional pattern that was both clearly distinct from other left PMd modules and any module on the right hemisphere. In particular, this region showed specific functional connectivity with the ventrolateral and dorsomedial prefrontal cortex, as well as with inferior parietal lobule. Accordingly, this module was integrated within a broader, rather prefrontal subregion in the parcellation of Glasser et al. (2016). In the present study, activity in the rostro-ventral left PMd was associated with tasks related to explicit longterm memory, object/scenes imagination, and deception paradigms. This pattern of functional interaction with higher associative regions and engagement in abstract behavioral functions suggests that this region, at the transition between left prefrontal cortex and left ventral PMd, may be involved in deriving mental abstractions from one's current ongoing situation/environment. Such abstraction involves reference to a different time frame (required for long-term memory retrieval), a different spatial frame (required for scenes imagination), and a different mental frame (required for deception). In line with whole-brain parcellation, there was no clear evidence for such rostro-ventral subregion in our right PMd parcellation suggesting that the right and left PMd are differently functionally characterized. To further confirm this hypothesis, we mirrored this module in the right hemisphere and examined its behavioral functional characterization (the results are reported in Supplementary Material, Figure S16). In this right hemisphere VOI, we did not find any significant relationship with the abstract functions evidenced in the left rostro-ventral module. Instead, this rostro-ventral part of the right PMd showed a similar behavioral characterization to the more superior rostral right PMd subregion. Therefore, we suggest that abstract functions are more predominantly represented in the posterior part of prefrontal cortex/anterior part of premotor cortex in the left hemisphere than in the right hemisphere.

\subsection{Conclusion}

In conclusion, for the first time, we described a parcellation, using a multimodal approach, of the left premotor cortex into five robust modules. The reliability of this approach was demonstrated by comparing the defined modules with previous coarser parcellations and the behavioral relevance of our modules was evidenced by robust functional characterization. The caudal, central and rostral left PMd modules confirmed the rostro-caudal organization reflecting a cognitive-motor gradient previously highlighted in the right PMd (Genon et al. 2016) and the frontal lobes as a whole (Yeo et al. 2011a; Glasser et al. 2016). Furthermore, our analysis suggested a left inferior PMd subregion involved in eye-field functions and thus 


\section{Genon et al.}

mirroring a right premotor eye-field (Genon et al. 2016), but also engaged in language functions and overlapping with Area 55b (Glasser et al., 2016). Finally, our multimodal parcellation also revealed a specific module in the rostro-ventral subregion supporting abstract cognitive functions, not evidenced in the corresponding PMd region in the right hemisphere.

\section{Funding}

This study was supported by the Deutsche Forschungsgemeinschaft (DFG, EI 816/4-1, LA 3071/3-1, GE 2835/1-1), the National Institute of Mental Health (R01-MH074457), the Helmholtz Portfolio Theme "Supercomputing and Modelling for the Human Brain", the European Union's Horizon 2020 Research and Innovation Programme under Grant Agreement No. 7202070 (HBP SGA1) and the National Institute of Mental Health (R01MH074457) 
Genon et al.

\section{$\underline{\text { References: }}$}

Abe M, Hanakawa T. 2009. Functional coupling underlying motor and cognitive functions of the dorsal premotor cortex. Behavioural brain research 198:13-23.

Amiez C, Petrides M. 2009. Anatomical organization of the eye fields in the human and non-human primate frontal cortex. Progress in neurobiology 89:220-230.

Amunts K, Schleicher A, Bürgel U, Mohlberg H, Uylings H, Zilles K. 1999. Broca's region revisited: cytoarchitecture and intersubject variability. Journal of Comparative Neurology 412:319-341.

Badre D. 2008. Cognitive control, hierarchy, and the rostro-caudal organization of the frontal lobes. Trends in cognitive sciences 12:193-200.

Badre D, D'Esposito M. 2009. Is the rostro-caudal axis of the frontal lobe hierarchical? Nature reviews Neuroscience 10:659-669.

Bahlmann J, Blumenfeld RS, D'Esposito M. 2015. The Rostro-Caudal Axis of Frontal Cortex Is Sensitive to the Domain of Stimulus Information. Cereb Cortex 25:1815-1826.

Behrens T, Berg HJ, Jbabdi S, Rushworth M, Woolrich M. 2007. Probabilistic diffusion tractography with multiple fibre orientations: What can we gain? NeuroImage 34:144155.

Behrens TE, Woolrich MW, Jenkinson M, Johansen-Berg H, Nunes RG, Clare S, Matthews PM, Brady JM, Smith SM. 2003a. Characterization and propagation of uncertainty in diffusion-weighted MR imaging. Magnetic resonance in medicine 50:1077-1088.

Behrens TEJ, Johansen-Berg H, Woolrich MW, Smith SM, Wheeler-Kingshott CAM, Boulby PA, Barker GJ, Sillery EL, Sheehan K, Ciccarelli O. 2003b. Non-invasive mapping of connections between human thalamus and cortex using diffusion imaging. Nature neuroscience 6:750-757.

Bellec P, Rosa-Neto P, Lyttelton OC, Benali H, Evans AC. 2010. Multi-level bootstrap analysis of stable clusters in resting-state fMRI. NeuroImage 51:1126-1139.

Birn RM. 2012. The role of physiological noise in resting-state functional connectivity. NeuroImage 62:864-870.

Boussaoud D. 2001. Attention versus intention in the primate premotor cortex. NeuroImage 14:S40-S45.

Boussaoud D, di Pellegrino G, Wise SP. 1995. Frontal lobe mechanisms subserving vision-for-action versus vision-for-perception. Behav Brain Res 72:1-15.

Burgess GC, Kandala S, Nolan D, Laumann TO, Power JD, Adeyemo B, Harms MP, Petersen SE, Barch DM. 2016. Evaluation of Denoising Strategies to Address MotionCorrelated Artifacts in Resting-State Functional Magnetic Resonance Imaging Data from the Human Connectome Project. Brain connectivity. 
Genon et al.

Caspers S, Geyer S, Schleicher A, Mohlberg H, Amunts K, Zilles K. 2006. The human inferior parietal cortex: cytoarchitectonic parcellation and interindividual variability. NeuroImage 33:430-448.

Caspers S, Zilles K, Laird AR, Eickhoff SB. 2010. ALE meta-analysis of action observation and imitation in the human brain. NeuroImage 50:1148-1167.

Cieslik EC, Seidler I, Laird AR, Fox PT, Eickhoff SB. 2016. Different involvement of subregions within dorsal premotor and medial frontal cortex for pro- and antisaccades. Neuroscience and biobehavioral reviews 68:256-269.

Cieslik EC, Zilles K, Caspers S, Roski C, Kellermann TS, Jakobs O, Langner R, Laird AR, Fox PT, Eickhoff SB. 2013. Is There "One" DLPFC in Cognitive Action Control? Evidence for Heterogeneity From Co-Activation-Based Parcellation. Cereb Cortex 23:2677-2689.

Clos M, Amunts K, Laird AR, Fox PT, Eickhoff SB. 2013. Tackling the multifunctional nature of Broca's region meta-analytically: co-activation-based parcellation of area 44 . NeuroImage 83:174-188.

De Schotten MT, Dell'Acqua F, Forkel SJ, Simmons A, Vergani F, Murphy DG, Catani M. 2011. A lateralized brain network for visuospatial attention. Nature neuroscience $14: 1245-1246$

Diedrichsen J, Balsters JH, Flavell J, Cussans E, Ramnani N. 2009. A probabilistic MR atlas of the human cerebellum. NeuroImage 46:39-46.

Eickhoff SB, Amunts K, Mohlberg H, Zilles K. 2006. The human parietal operculum. II. Stereotaxic maps and correlation with functional imaging results. Cereb Cortex 16:268279.

Eickhoff SB, Bzdok D, Laird AR, Kurth F, Fox PT. 2012. Activation likelihood estimation meta-analysis revisited. NeuroImage 59:2349-2361.

Eickhoff SB, Bzdok D, Laird AR, Roski C, Caspers S, Zilles K, Fox PT. 2011. Co-activation patterns distinguish cortical modules, their connectivity and functional differentiation. NeuroImage 57:938-949.

Eickhoff SB, Stephan KE, Mohlberg H, Grefkes C, Fink GR, Amunts K, Zilles K. 2005. A new SPM toolbox for combining probabilistic cytoarchitectonic maps and functional imaging data. NeuroImage 25:1325-1335.

Eickhoff SB, Thirion B, Varoquaux G, Bzdok D. 2015. Connectivity-based parcellation: Critique and implications. Human brain mapping 36:4771-4792.

Foster BL, He BJ, Honey CJ, Jerbi K, Maier A, Saalmann YB. 2016. Spontaneous Neural Dynamics and Multi-scale Network Organization. Frontiers in systems neuroscience 10:7.

Genon S, Li H, Fan L, Muller VI, Cieslik EC, Hoffstaedter F, Reid AT, Langner R, Grefkes C, Fox PT, Moebus S, Caspers S, Amunts K, Jiang T, Eickhoff SB. 2016. The Right Dorsal Premotor Mosaic: Organization, Functions, and Connectivity. Cereb Cortex. 
Genon et al.

Geyer S, Ledberg A, Schleicher A, Kinomura S, Schormann T, Burgel U, Klingberg T, Larsson J, Zilles K, Roland PE. 1996. Two different areas within the primary motor cortex of man. Nature 382:805-807.

Geyer S, Matelli M, Luppino G, Zilles K. 2000. Functional neuroanatomy of the primate isocortical motor system. Anatomy and embryology 202:443-474.

Geyer S, Schleicher A, Zilles K. 1999. Areas 3a, 3b, and 1 of human primary somatosensory cortex. NeuroImage 10:63-83.

Glasser M, Coalson T, Robinson E, Hacker C, Harwell J, Yacoub E, Ugurbil K, Anderson J, Beckmann C, Jenkinson M. 2016. A Multi-modal parcellation of human cerebral cortex. Nature.

Grefkes C, Geyer S, Schormann T, Roland P, Zilles K. 2001. Human somatosensory area 2: observer-independent cytoarchitectonic mapping, interindividual variability, and population map. NeuroImage 14:617-631.

Griffanti L, Salimi-Khorshidi G, Beckmann CF, Auerbach EJ, Douaud G, Sexton CE, Zsoldos E, Ebmeier KP, Filippini N, Mackay CE, Moeller S, Xu J, Yacoub E, Baselli G, Ugurbil K, Miller KL, Smith SM. 2014. ICA-based artefact removal and accelerated fMRI acquisition for improved resting state network imaging. NeuroImage 95:232-247.

Grosbras MH, Beaton S, Eickhoff SB. 2012. Brain regions involved in human movement perception: a quantitative voxel-based meta-analysis. Human brain mapping 33:431454.

Gross CG. 1994. How inferior temporal cortex became a visual area. Cerebral cortex 4:455-469.

Hanakawa T. 2011. Rostral premotor cortex as a gateway between motor and cognitive networks. Neuroscience research 70:144-154.

Hardwick RM, Rottschy C, Miall RC, Eickhoff SB. 2013. A quantitative meta-analysis and review of motor learning in the human brain. NeuroImage 67:283-297.

Hoshi E, Tanji J. 2007. Distinctions between dorsal and ventral premotor areas: anatomical connectivity and functional properties. Current opinion in neurobiology $17: 234-242$.

Kantak SS, Stinear JW, Buch ER, Cohen LG. 2012. Rewiring the brain: potential role of the premotor cortex in motor control, learning, and recovery of function following brain injury. Neurorehabilitation and neural repair 26:282-292.

Kell CA, Morillon B, Kouneiher F, Giraud A-L. 2010. Lateralization of speech production starts in sensory cortices-a possible sensory origin of cerebral left dominance for speech. Cerebral Cortex:bhq167.

Koechlin E, Summerfield C. 2007. An information theoretical approach to prefrontal executive function. Trends in cognitive sciences 11:229-235. 
Genon et al.

Laird AR, Eickhoff SB, Kurth F, Fox PM, Uecker AM, Turner JA, Robinson JL, Lancaster JL, Fox PT. 2009. ALE meta-analysis workflows via the brainmap database: progress towards a probabilistic functional brain atlas. Frontiers in neuroinformatics 3 .

Langleben DD, Schroeder L, Maldjian JA, Gur RC, McDonald S, Ragland JD, O'Brien CP, Childress AR. 2002. Brain activity during simulated deception: an event-related functional magnetic resonance study. NeuroImage 15:727-732.

Langner R, Eickhoff SB. 2013. Sustaining attention to simple tasks: a meta-analytic review of the neural mechanisms of vigilant attention. Psychological bulletin 139:870900.

Laumann TO, Gordon EM, Adeyemo B, Snyder AZ, Joo SJ, Chen MY, Gilmore AW, McDermott KB, Nelson SM, Dosenbach NU, Schlaggar BL, Mumford JA, Poldrack RA, Petersen SE. 2015. Functional System and Areal Organization of a Highly Sampled Individual Human Brain. Neuron 87:657-670.

Lebedev MA, Wise SP. 2001. Tuning for the orientation of spatial attention in dorsal premotor cortex. European Journal of Neuroscience 13:1002-1008.

Luppino G, Rozzi S, Calzavara R, Matelli M. 2003. Prefrontal and agranular cingulate projections to the dorsal premotor areas F2 and F7 in the macaque monkey. The European journal of neuroscience 17:559-578.

Matelli M, Luppino G, Rizzolatti G. 1985. Patterns of cytochrome oxidase activity in the frontal agranular cortex of the macaque monkey. Behavioural brain research 18:125136.

Matelli M, Luppino G, Rizzolatti G. 1991. Architecture of superior and mesial area 6 and the adjacent cingulate cortex in the macaque monkey. Journal of Comparative Neurology 311:445-462.

Morosan P, Schleicher A, Amunts K, Zilles K. 2005. Multimodal architectonic mapping of human superior temporal gyrus. Anat Embryol (Berl) 210:401-406.

Murphy K, Birn RM, Bandettini PA. 2013. Resting-state fMRI confounds and cleanup. NeuroImage 80:349-359.

Nakayama Y, Yamagata T, Hoshi E. 2016. Rostrocaudal functional gradient among the pre-dorsal premotor cortex, dorsal premotor cortex and primary motor cortex in goaldirected motor behaviour. The European journal of neuroscience 43:1569-1589.

Orban P, Doyon J, Petrides M, Mennes M, Hoge R, Bellec P. 2015. The Richness of TaskEvoked Hemodynamic Responses Defines a Pseudohierarchy of Functionally Meaningful Brain Networks. Cereb Cortex 25:2658-2669.

Picard N, Strick PL. 2001. Imaging the premotor areas. Current opinion in neurobiology 11:663-672.

Reid AT, Bzdok D, Genon S, Langner R, Muller VI, Eickhoff CR, Hoffstaedter F, Cieslik EC, Fox PT, Laird AR, Amunts K, Caspers S, Eickhoff SB. 2016. ANIMA: A data-sharing initiative for neuroimaging meta-analyses. NeuroImage 124:1245-1253. 
Genon et al.

Rizzolatti G, Luppino G. 2001. The Cortical Motor System. Neuron 31:889-901.

Rothstein HR, Sutton AJ, Borenstein M. 2006. Publication Bias in Meta-Analysis. In. Publication Bias in Meta-Analysis John Wiley \& Sons, Ltd p 1-7.

Rottschy C, Caspers S, Roski C, Reetz K, Dogan I, Schulz JB, Zilles K, Laird AR, Fox PT, Eickhoff SB. 2013. Differentiated parietal connectivity of frontal regions for "what" and "where" memory. Brain structure \& function 218:1551-1567.

Rottschy C, Langner R, Dogan I, Reetz K, Laird AR, Schulz JB, Fox PT, Eickhoff SB. 2012. Modelling neural correlates of working memory: a coordinate-based meta-analysis. NeuroImage 60:830-846.

Salimi-Khorshidi G, Douaud G, Beckmann CF, Glasser MF, Griffanti L, Smith SM. 2014. Automatic denoising of functional MRI data: combining independent component analysis and hierarchical fusion of classifiers. NeuroImage 90:449-468.

Scheperjans F, Eickhoff SB, Homke L, Mohlberg H, Hermann K, Amunts K, Zilles K. 2008. Probabilistic maps, morphometry, and variability of cytoarchitectonic areas in the human superior parietal cortex. Cereb Cortex 18:2141-2157.

Schubotz RI, Anwander A, Knösche TR, von Cramon DY, Tittgemeyer M. 2010. Anatomical and functional parcellation of the human lateral premotor cortex. NeuroImage 50:396-408.

Sigl B, Caspers S, Mohlberg H, Cieslik EC, Eickhoff SB, Amunts K. 2016. The human dorsal premotor cortex - cytoarchitecture, maps and function. Proceedings of the OHBM 2016 Annual Meeting.

Van Essen DC, Jbabdi S, Sotiropoulos SN, Chen C, Dikranian K, Coalson T, Harwell J, Behrens TE, Glasser MF. 2013. Mapping connections in humans and nonhuman primates: aspirations and challenges for diffusion imaging. Diffusion MRI, 2nd edition (eds Johansen-Berg, H \& Behrens, TEJ):337-358.

Vossel S, Geng JJ, Fink GR. 2014. Dorsal and ventral attention systems distinct neural circuits but collaborative roles. The Neuroscientist 20:150-159.

Wager TD, Jonides J, Reading S. 2004. Neuroimaging studies of shifting attention: a metaanalysis. NeuroImage 22:1679-1693.

Yeo BT, Krienen FM, Sepulcre J, Sabuncu MR, Lashkari D, Hollinshead M, Roffman JL, Smoller JW, Zöllei L, Polimeni JR. 2011a. The organization of the human cerebral cortex estimated by intrinsic functional connectivity. Journal of neurophysiology 106:11251165.

Yeo BT, Krienen FM, Sepulcre J, Sabuncu MR, Lashkari D, Hollinshead M, Roffman JL, Smoller JW, Zollei L, Polimeni JR, Fischl B, Liu H, Buckner RL. 2011b. The organization of the human cerebral cortex estimated by intrinsic functional connectivity. J Neurophysiol 106:1125-1165.

Yeo BTT, Krienen FM, Sepulcre J, Sabuncu MR, Lashkari D, Hollinshead M, Roffman JL, Smoller JW, Zöllei L, Polimeni JR. 2011c. The organization of the human cerebral cortex 
Genon et al.

estimated by intrinsic functional connectivity. Journal of neurophysiology 106:11251165. 

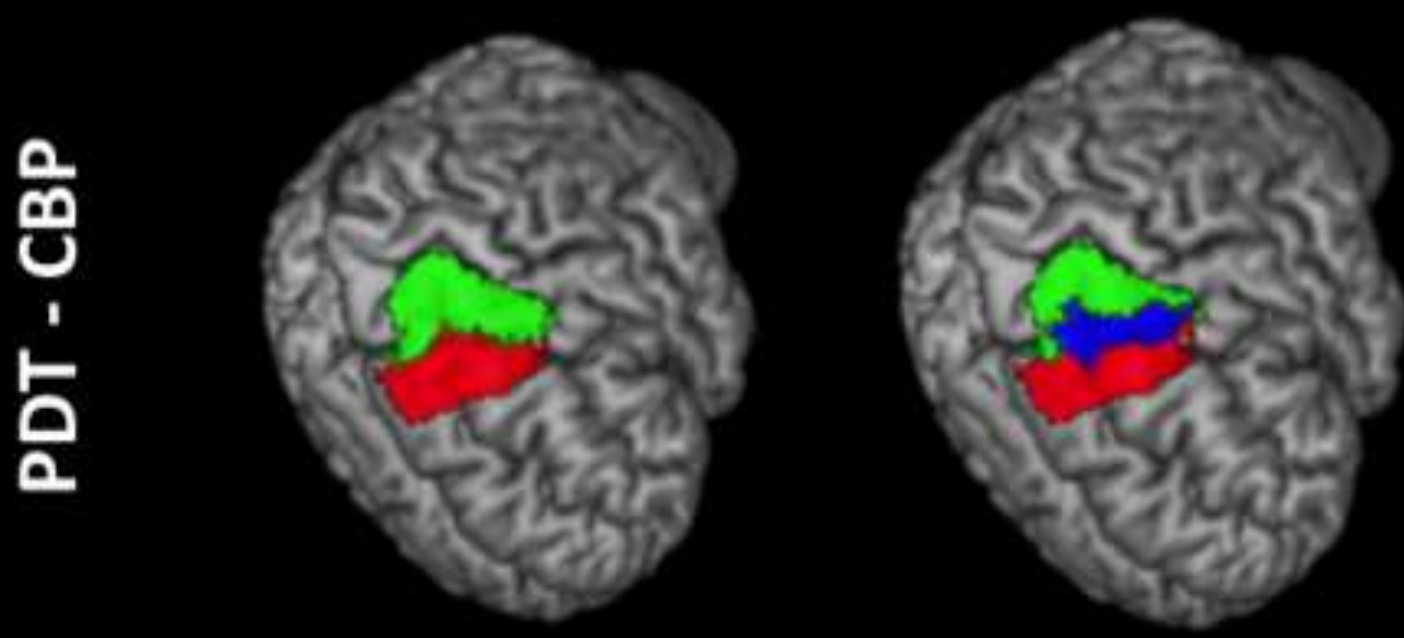

$\frac{a}{6}$
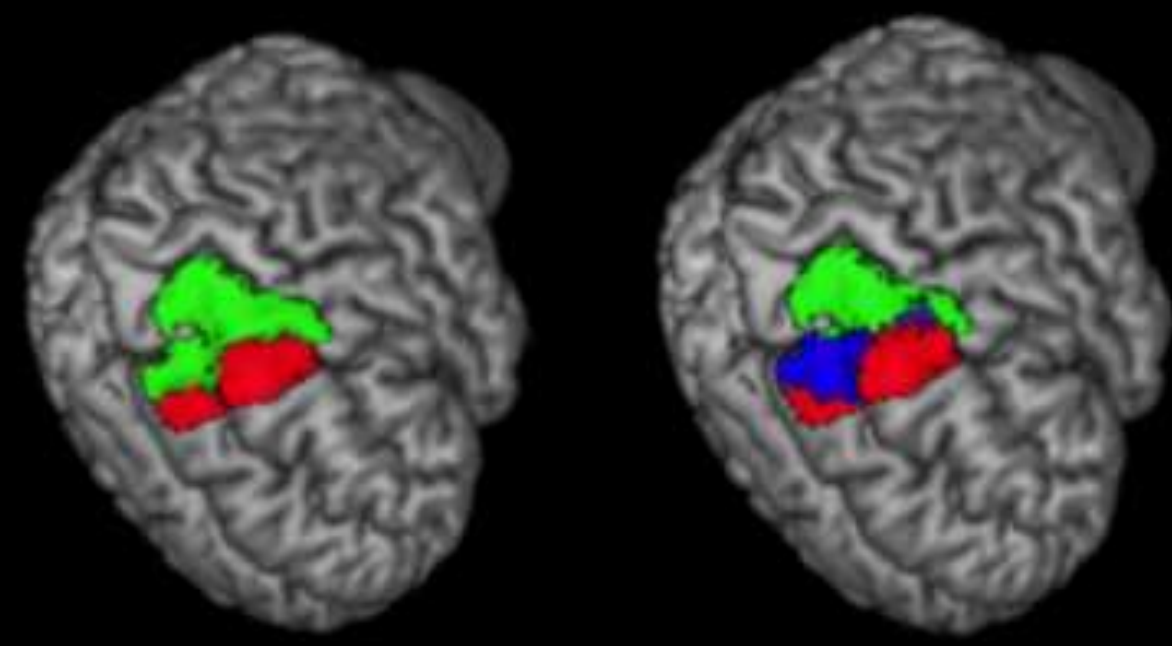

a

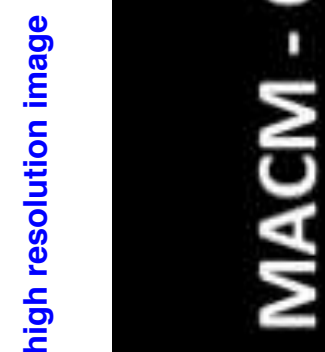

1

$\frac{1}{4}$

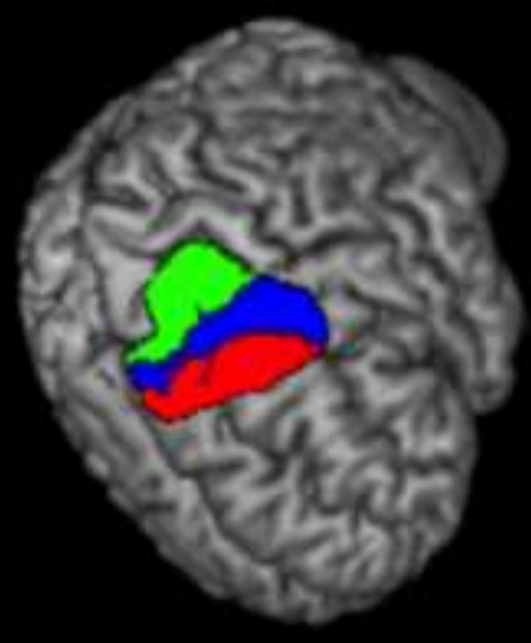

厂

익

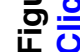


Figure_2

Click here to download high resolution image

MACM - CBP

RSFC - CBP

PDT - CBP

k4
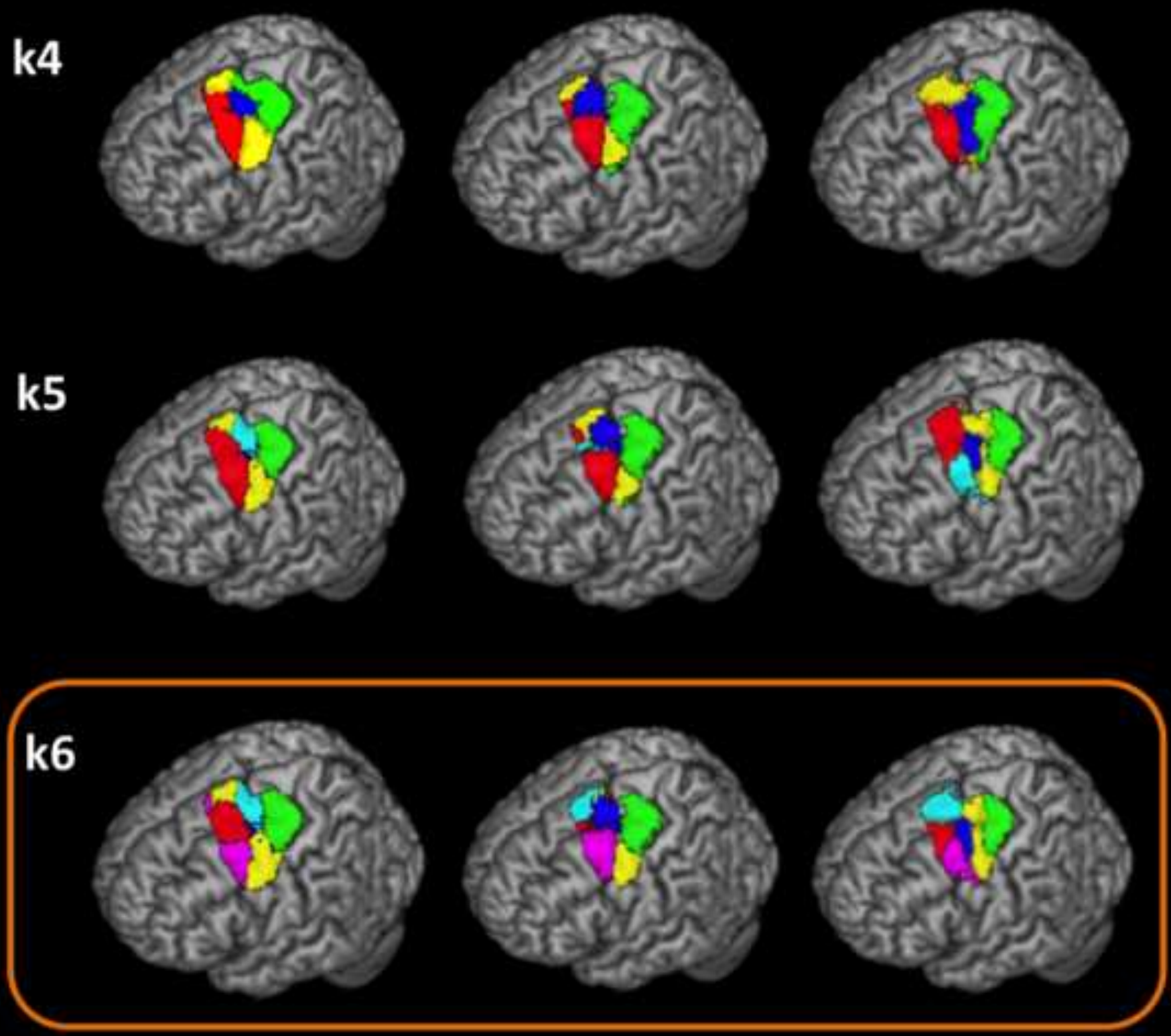

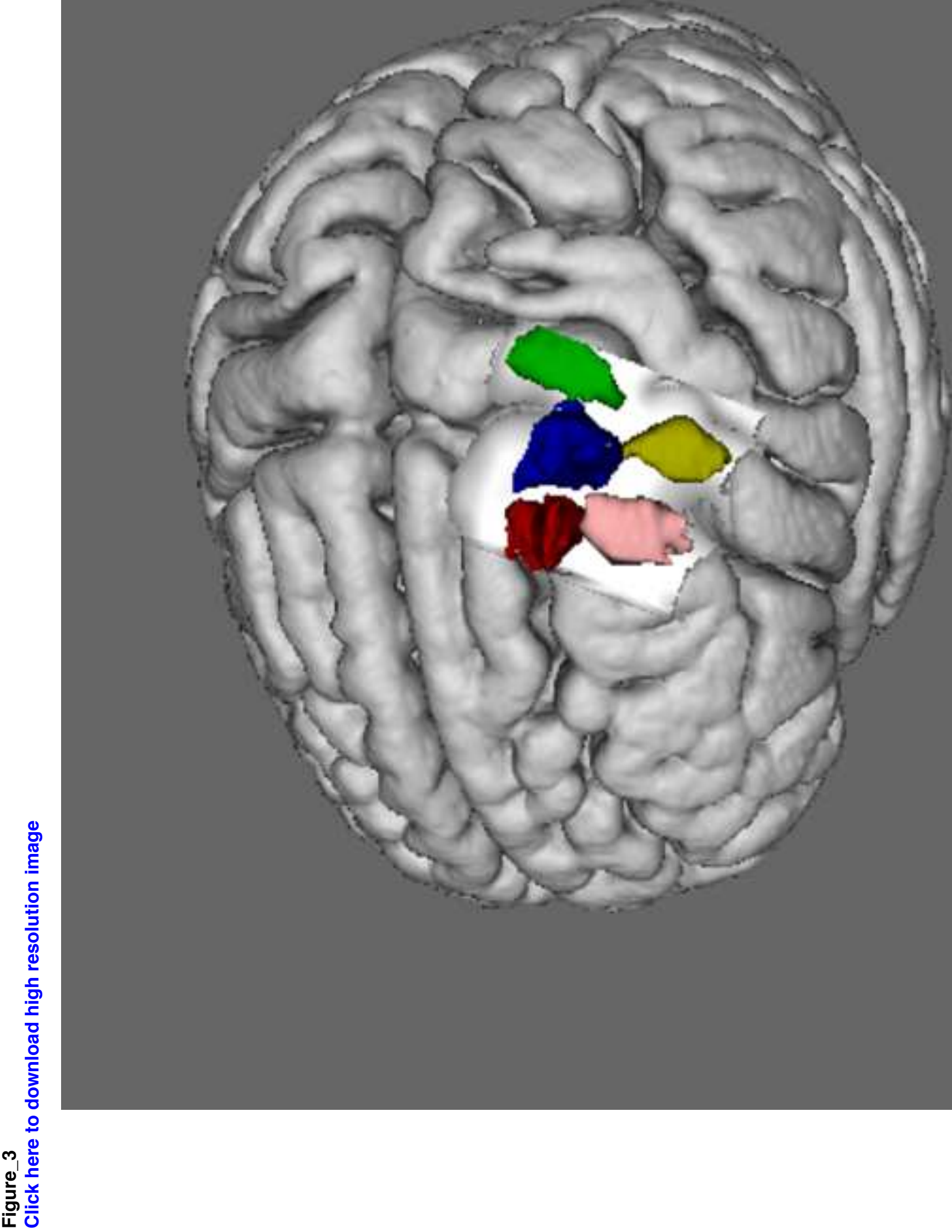

๓

인

(5) 
Figure_4

Click here to download high resolution image
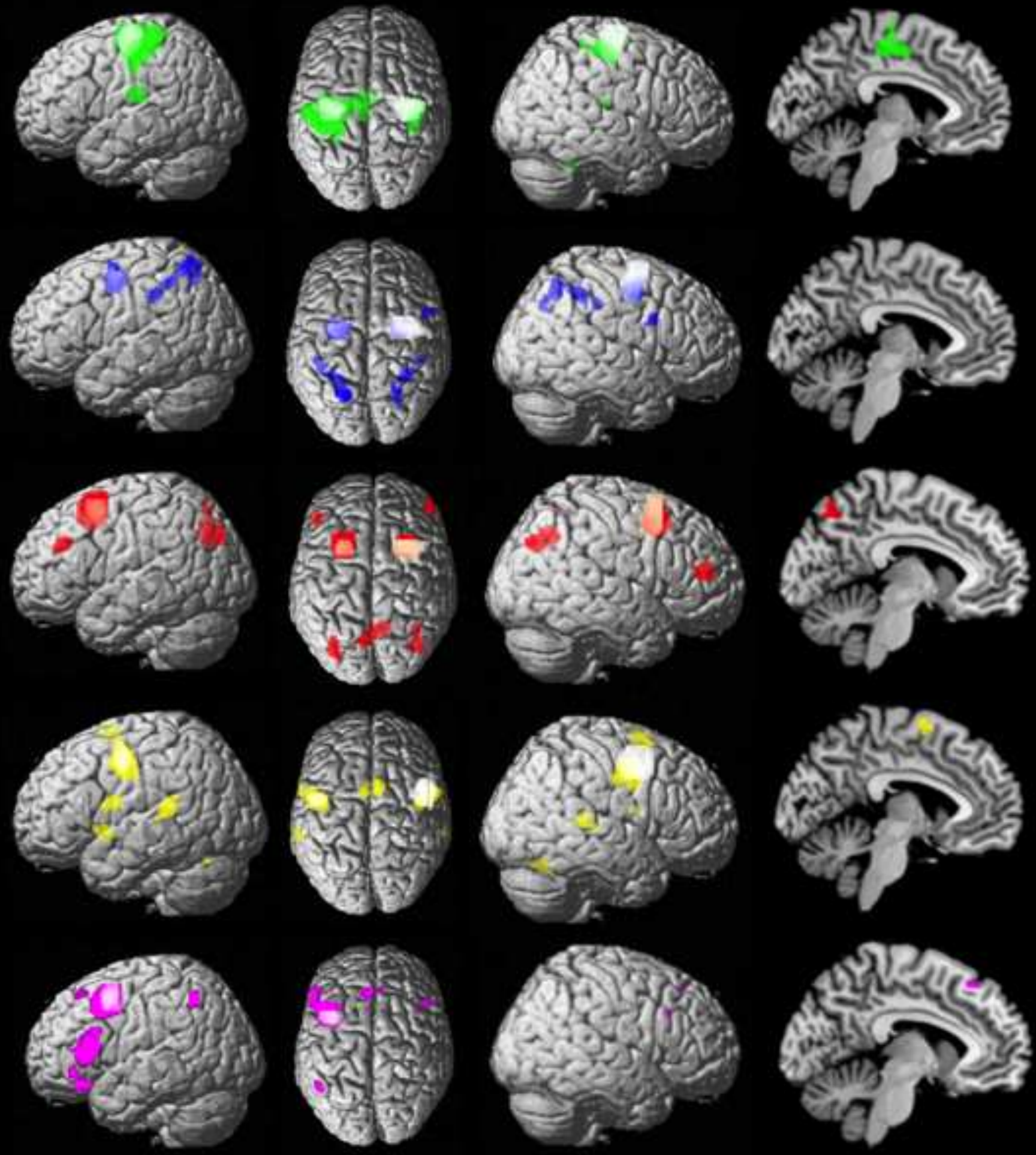
Figure_5

Click here to download 9. Figure: FighauededednctDeEpantral

Behavioral Domains

Perception Somestesis

Action execution

Motor learning

Attention

Spatial cognition

Action imagination

Motion vision

Working memory

Reasoning

Speech cognition

Speech execution

Music cognition

Explicit memory

\section{Paradigm Classes}

\section{TMS}

Flexion/extension

Grasping

Pointing

Tactile monitor discrimination Finger tapping

Sequenc recall/learning

Mental rotation

Imagined object/scene

Visuospatial attention

Visual Object identification

Saccades

Anti-saccades

Reading (overt)

Recitation/repetition

Tower of London

$\mathrm{N}$-back

Counting/calculation

Reasoning/problem solving

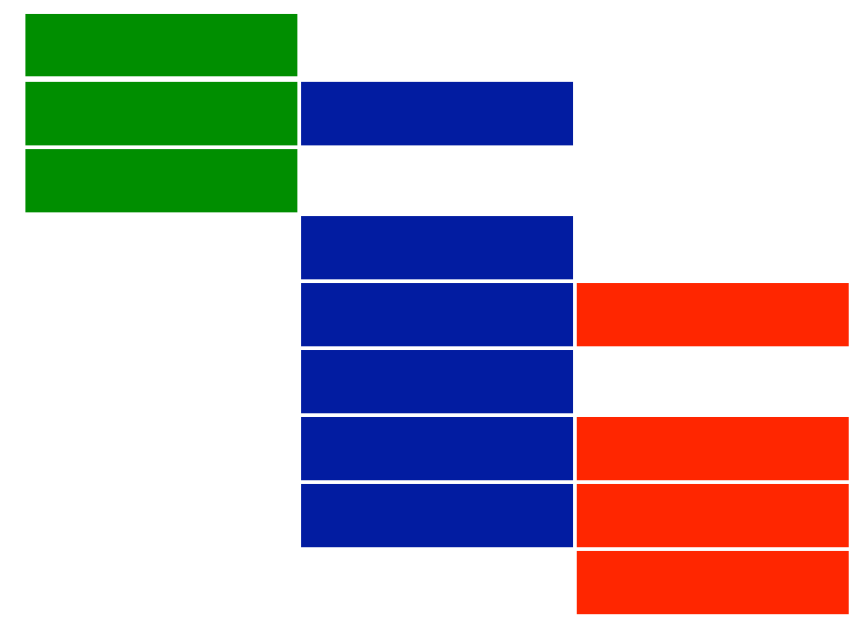

Deception
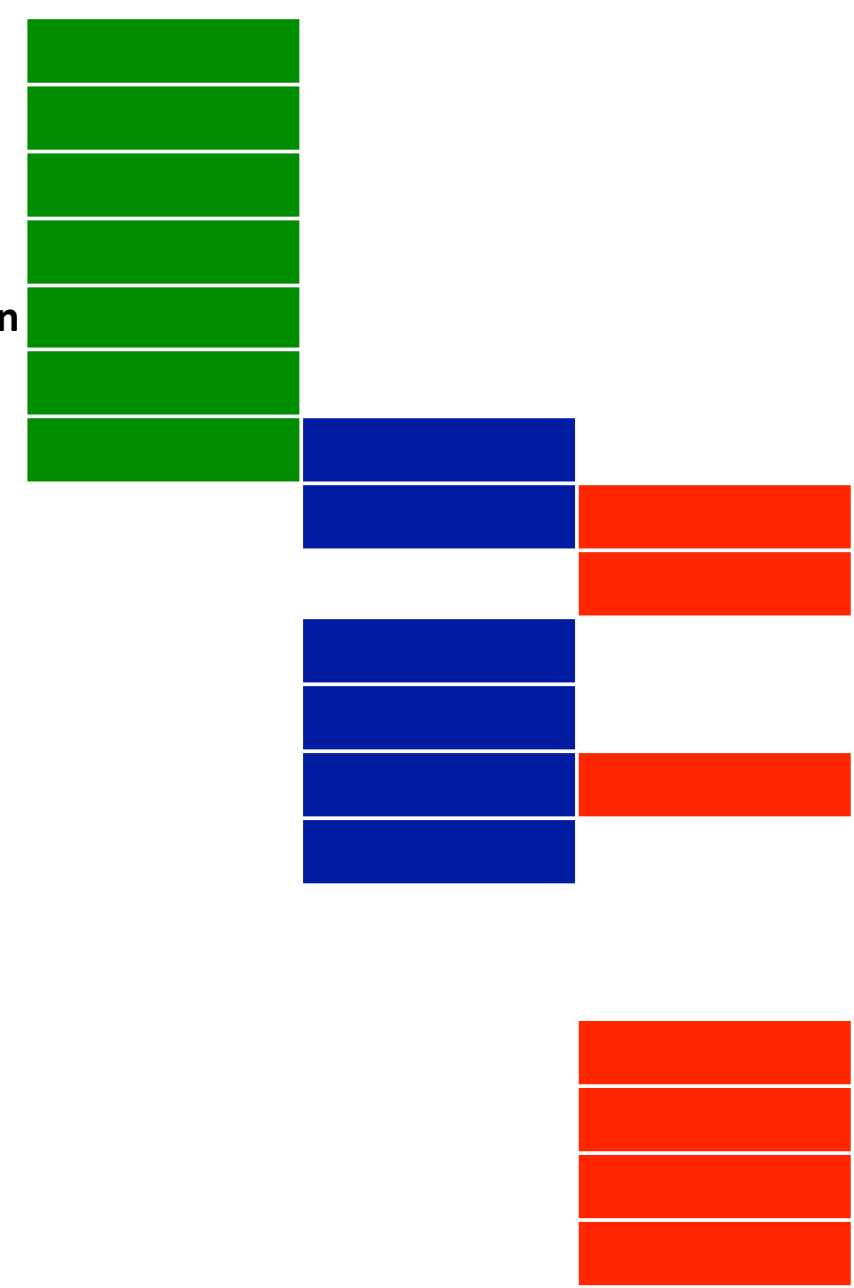


\section{Supplemental introduction}

Differential organization within the right and left PMd have been evidenced, albeit indirectly, by previous parcellation studies. For example, whole-brain organization according to intrinsic connectivity performed by Yeo et al. in 2011 and illustrated in Figure S1 revealed the broad definition of PMd as one of the brain regions showing the higher functional asymmetry. Whereas the posterior portion of the premotor cortex showed a similar engagement in the assumed sensori-motor network, the right and left anterior parts of the premotor cortex (posterior superior and middle frontal gyri) showed different functional coupling suggesting asymmetric functional gradient.

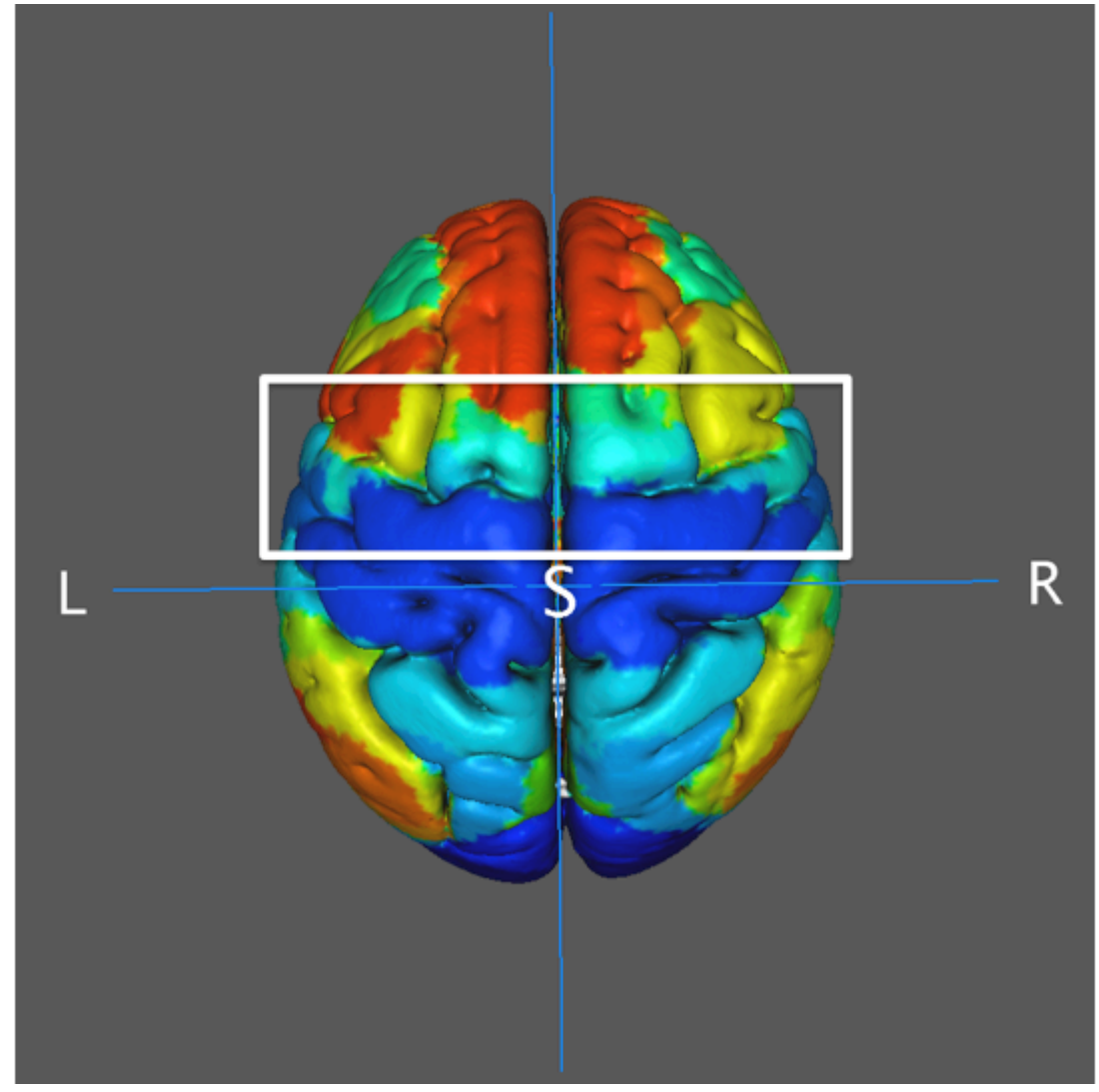

Figure S1. Cortical parcellation estimated by intrinsic functional connectivity from Yeo et al. (2011) mapped to volume (L, left, R, right, S, central sulcus; 17 networks map from https://surfer.nmr.mgh.harvard.edu/fswiki/CorticalParcellation_Yeo2011 displayed with Mango: http://ric.uthscsa.edu/mango/). 


\section{Supplemental methods}

\section{II.1. MACM-CBP: data-driven selection of the optimal filter range}

In line with previous MACM-CBP studies (Clos et al. 2013; Genon et al. 2016), the optimal filter range was chosen based on the consistency of each voxel's cluster assignment across the different filter sizes. The proportion of deviants (normalized within each cluster solution $\mathrm{k}$ ) and the derived weighted sum of the z-normalized number of deviants across clusters are illustrated in Figure S1. As already pointed out in previous MACM-CBP studies (Clos et al. 2013; Genon et al. 2016) parcellations based on small as well as large filter sizes yielded more deviants. The filter size range chosen (94 to 154) was based on the increase in the weighted sum (across all k) of the z-normalized number of deviants before and after these filter size values (cut off at $\mathrm{z}<-0.5$ : only those filter sizes were included where the number of deviants was at least half a standard deviation lower than the average number of deviants across all filter sizes).
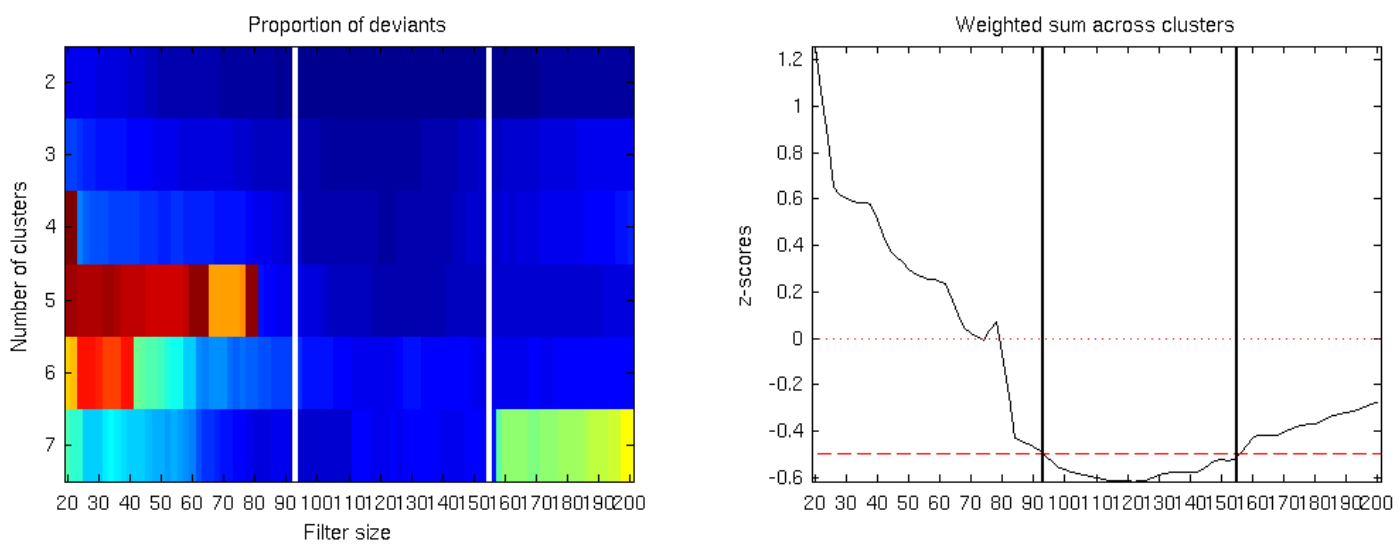

Figure S2. MACM-CBP: deviants and stability. z-scores on median-filtered deviants (normalized for k). The vertical lines specify the ultimately selected, most stable range of filter sizes (i.e., range with least deviants across k). Left: the proportion of deviants computed across filter sizes. Warm/cold colors indicate high/low numbers of deviants. Right: Maximum z- score of median-filtered deviants. 


\section{II.2. PDT-CBP: data-driven exclusion of surface voxels}

PDT generally focuses on voxels at GM/WM boundaries as tractography estimates deteriorates with spatial remoteness from the starting GM/WM boundaries voxels. To ensure successful PDT of PMd voxels, a spatial constraint can be introduced with an inclusive mask restricting PDT to voxels at GM/WM boundaries in each subject (e.g.: (Tomassini et al. 2007)). Nevertheless, in the present study, the definition of a group mask (for the 124 subjects) of GM/WM boundaries would have required relatively arbitrary selection of the spatial remoteness from the exact GM/WM boundaries at the group level. Therefore, we opted for a data-driven definition of the GM voxels whose spatial remoteness from WM boundaries prevented successful tractography. Accordingly, PDT-CBP on the whole left PMd VOI revealed a spurious cluster formed by surface voxels as illustrated in Figure S3 (880 voxels). Thus, these voxels were excluded from the left PMd VOI and PDT-CBP was then successfully run on the remaining VOI.

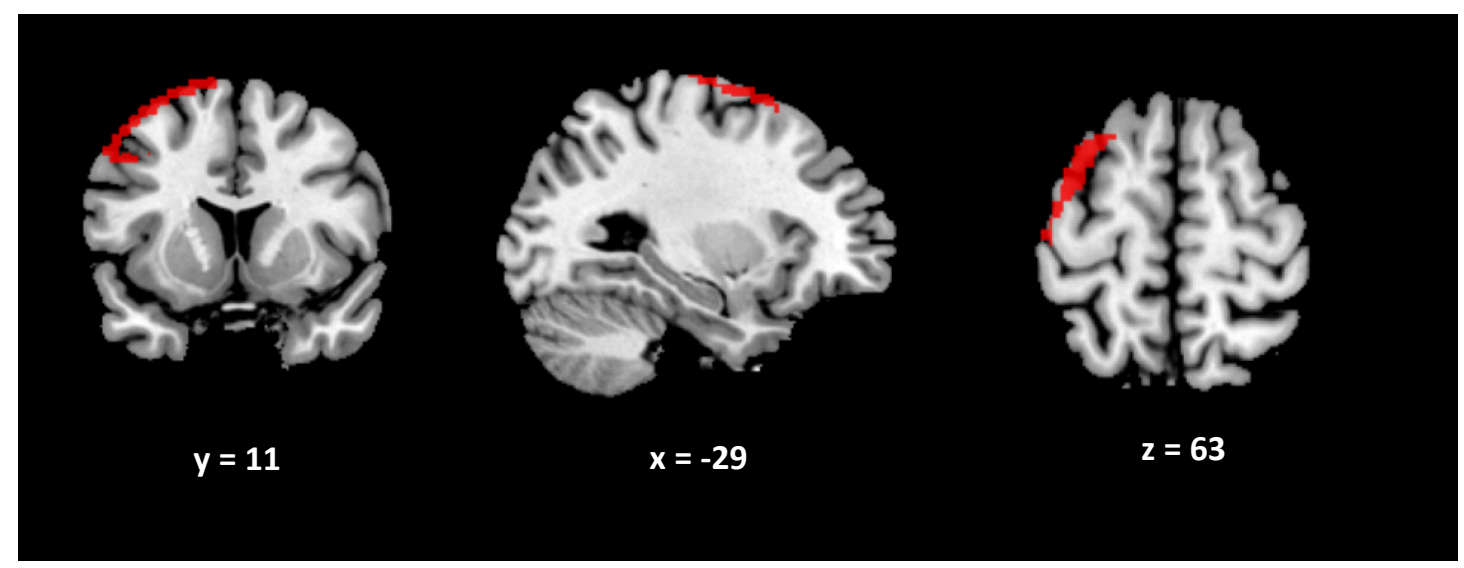

Figure S3. Emergence of a spurious cluster formed by surface voxels following PDT-CBP of the whole left PMd VOI. 


\section{Supplemental results}

\section{III.1. Parcellation stability}

We examined how the parcellations were supported by the data for the higher scales $(\mathrm{k}=4-6)$ independently for each CBP modality (MACM, RSFC and PDT) based on three criteria: the percentage of voxels not with parents, the change in intra/inter distance and the percentage of deviants. Of note, $\mathrm{k}=2$ and $\mathrm{k}=7$ are not strictly evaluated for these criteria as these $\mathrm{k}$ solutions are the borders of our $\mathrm{k}$ range and thus, the following cluster solution. Our four criteria are considered for guiding the choice of the cluster solution at higher resolution (i.e. choosing between $\mathrm{k}=4, \mathrm{k}=5$ and $\mathrm{k}=6$ ) below $\mathrm{k}=7$.

$M A C M-C B P$ : The hierarchy index decreased from $\mathrm{k}=5$ to $\mathrm{k}=6$ being lower than $5 \%$ at $\mathrm{k}=$ 6.The inter/intra cluster ratio increased from $\mathrm{k}=4$ to $\mathrm{k}=6$, while it decreased at subsequent solution suggesting $\mathrm{k}=6$ as a local optimum. In contrast, the silhouette value decreased from $\mathrm{k}=3$ to $\mathrm{k}=6$. Similarly, the percentage of deviants did not promote any cluster solution as it significantly increased from $\mathrm{k}=3$ to $\mathrm{k}=6$.

$R S F C-C B P$ : The percentage of voxels not with parents suggested two local optima across the cluster solution: $\mathrm{k}=4$ and $\mathrm{k}=6$. In contrast, both, the change in intra/inter distance ratio and the silhouette value did not promote a particular parcellation as it monotically decreased from $\mathrm{k}=3$ to $\mathrm{k}=7$. The percentage of deviants was also not informative as it showed significant increase from $\mathrm{k}=3$ to $\mathrm{k}=7$.

PDT-CBP: The hierarchy index showed a sharp decrease below $5 \%$ at $6 \mathrm{k}$. In contrast, and in line with RSFC-CBP, the examination of the change in intra/inter distance ratio did not promote a particular parcellation at higher scale as it decreased from $\mathrm{k}=3$ to $\mathrm{k}=7$. In contrast, the percentage of deviants across cluster solutions significantly increased from $\mathrm{k}=3$ to $\mathrm{k}=4$, stayed relatively stable to $\mathrm{k}=6$ and then significantly increased again beyond $\mathrm{k}=6$. 
MACM-CBP
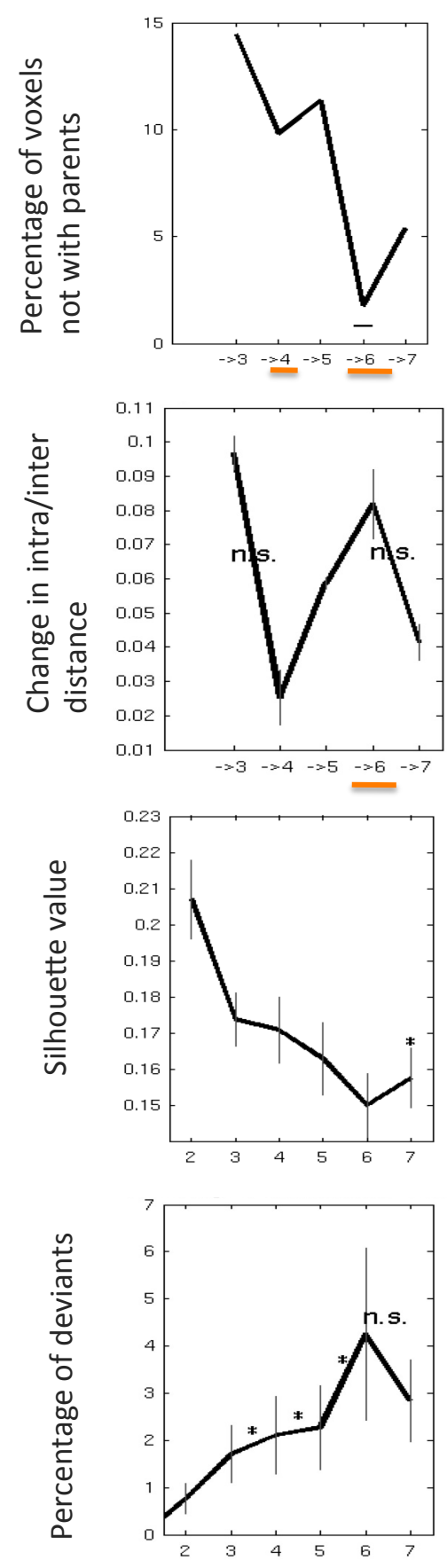

RSFC-CBP
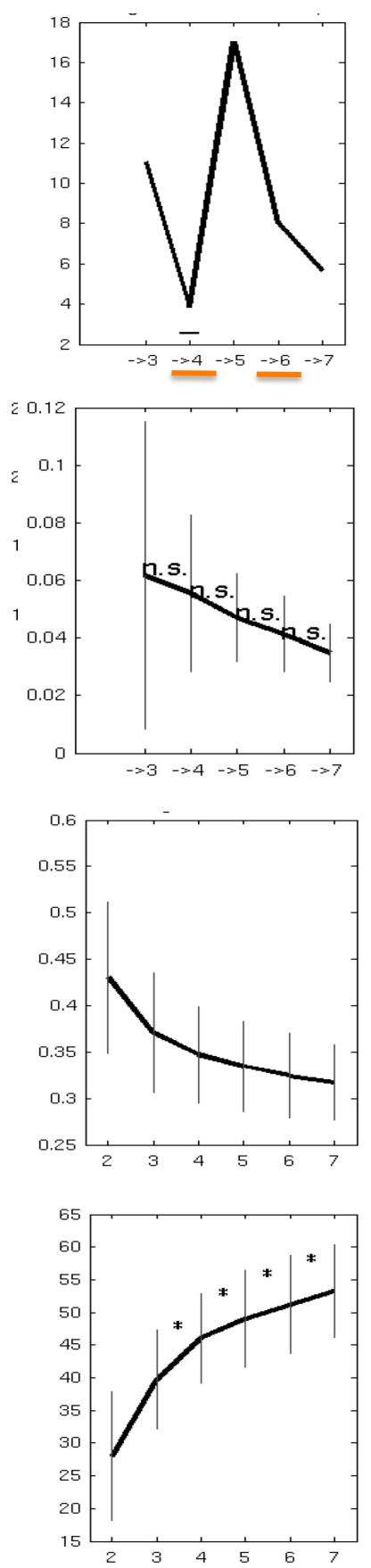

PDT-CBP
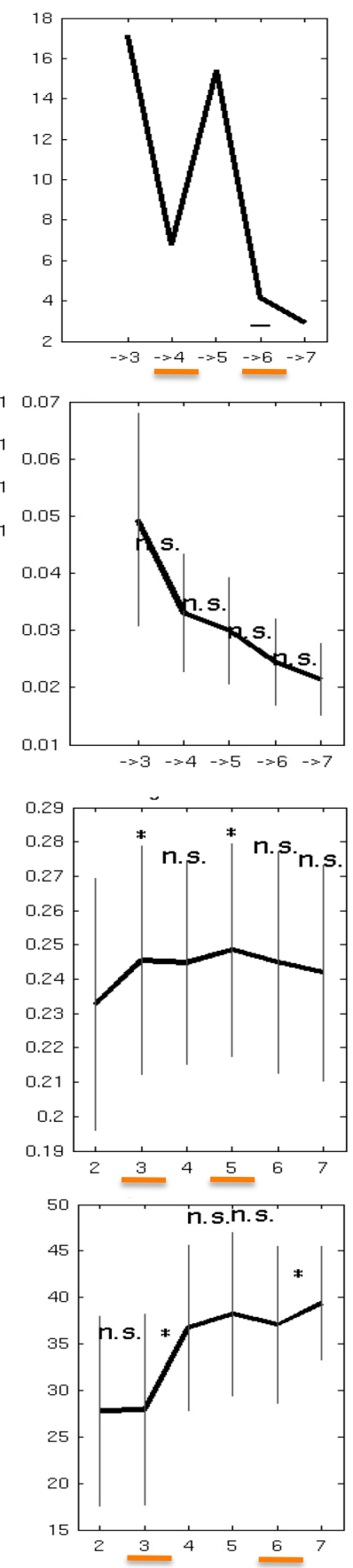

Figure S4. Cluster solution criteria. *Significant change in percentage of deviants. N.s. non significant change. 


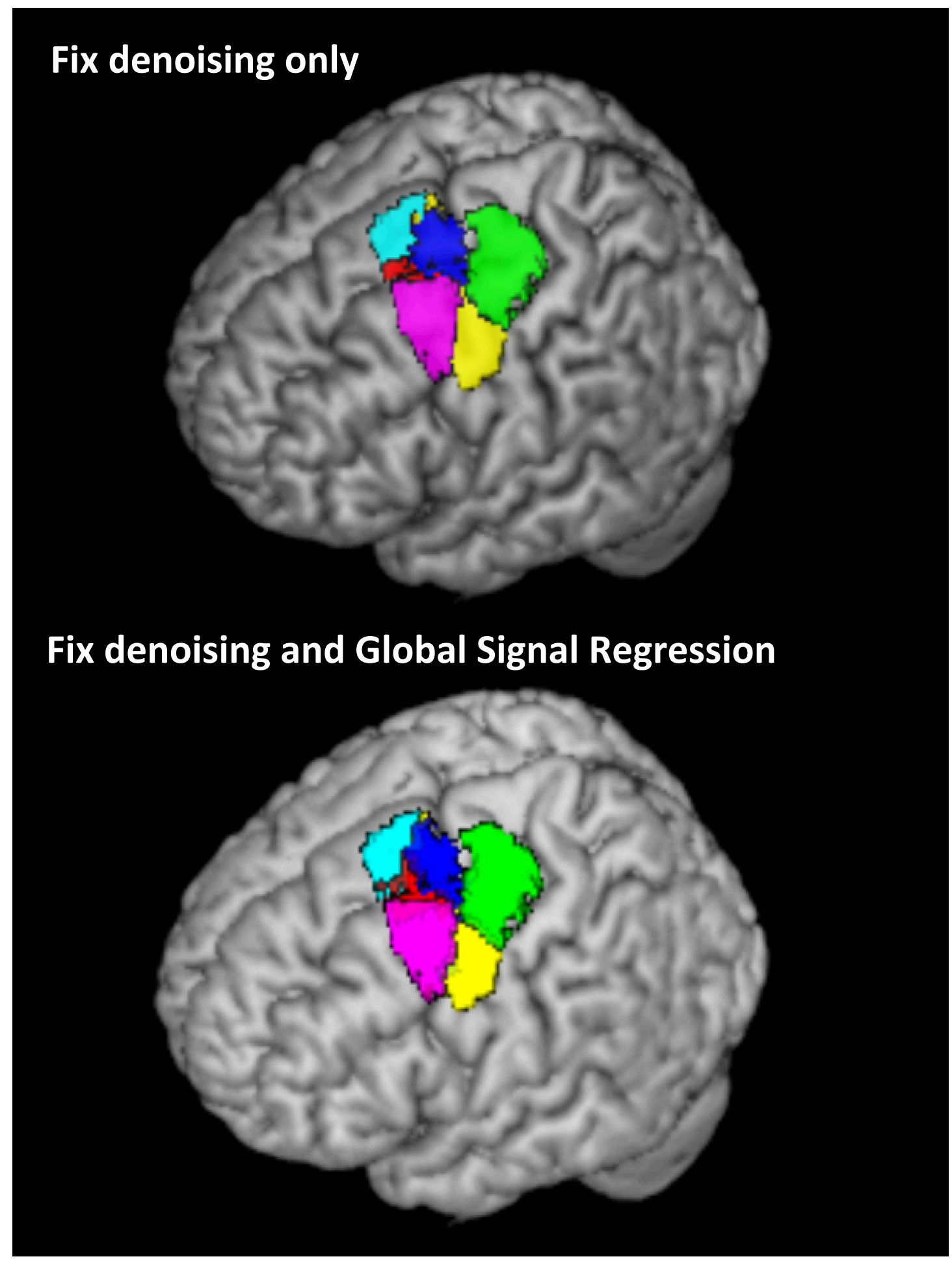

Figure S5. Six clusters-solution provided by RSFC-CBP following Fix denoising only and Fix denoising combined with linear Global Signal Regression (GSR). 


\section{NKI sample}

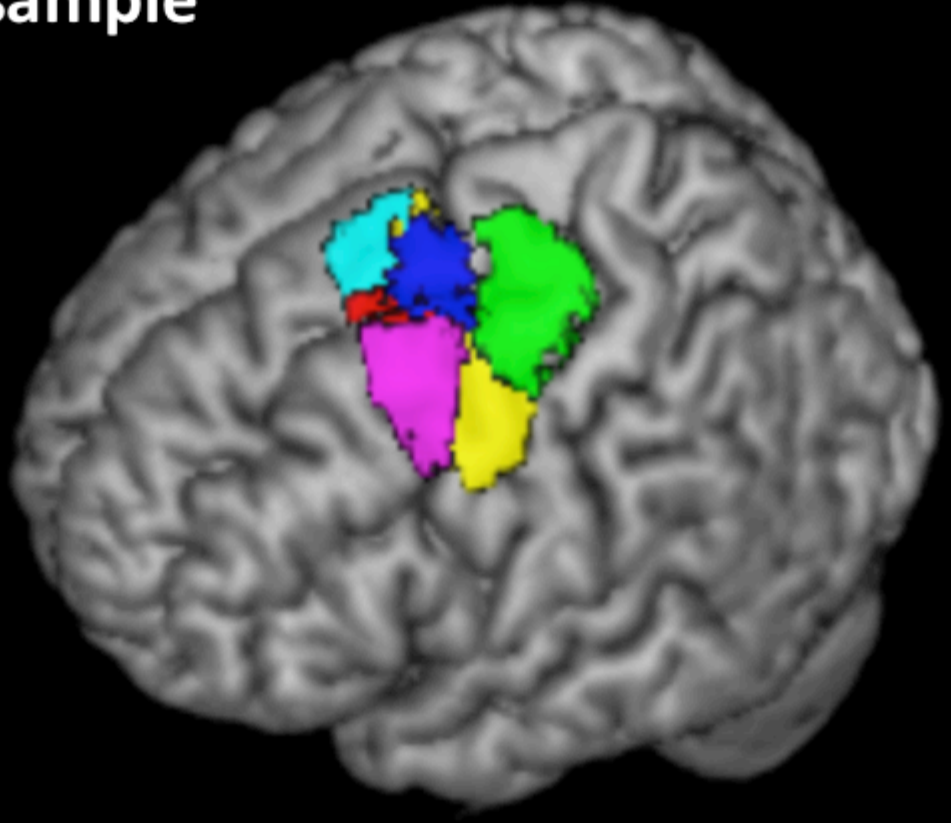

\section{HCP sample}

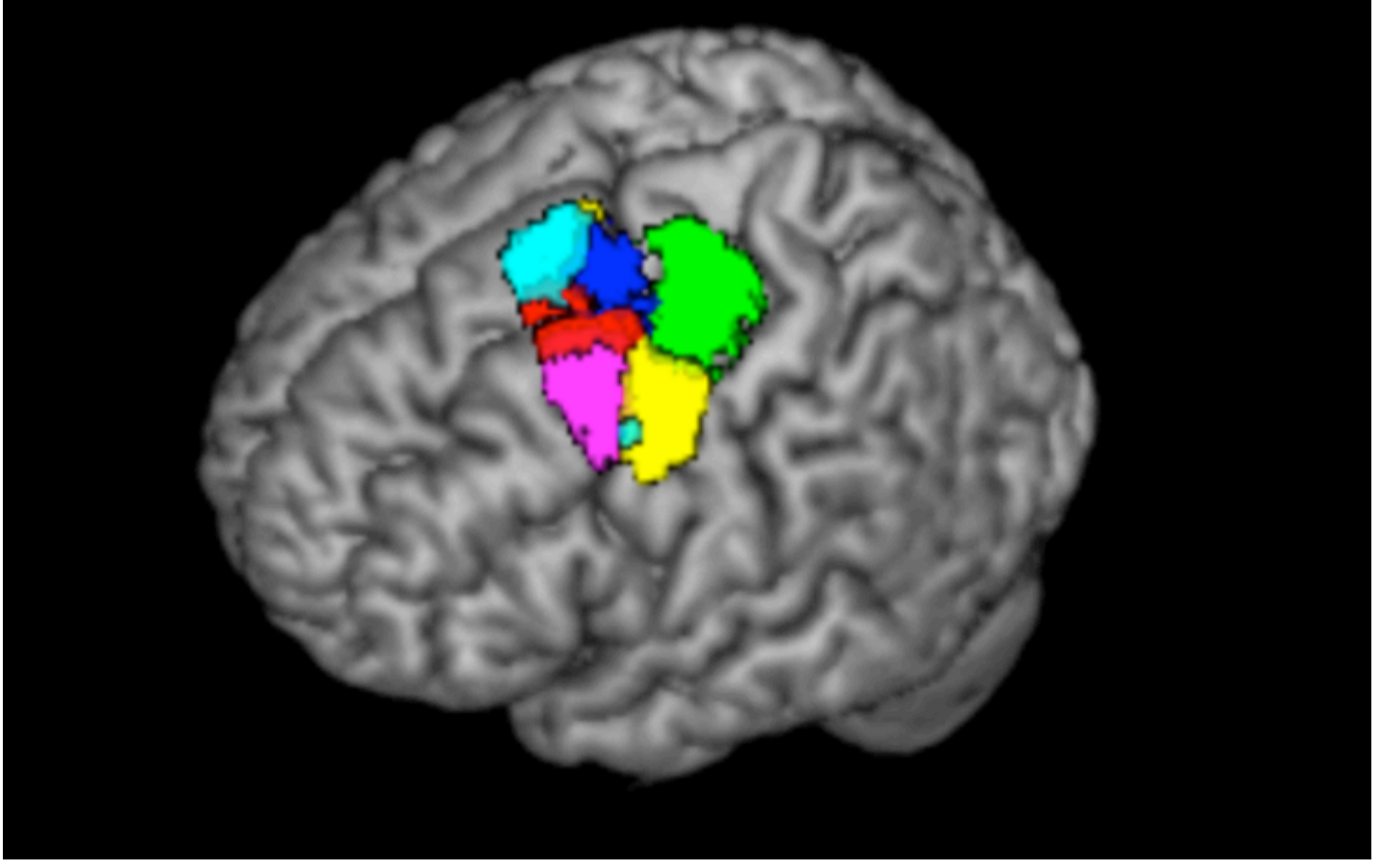

Figure S6. Six-clusters solution provided by RSFC-CBP on RS fMRI data of 124 healthy adults ( 84 females; mean age $46.56 \pm 17.56$ ) from the enhanced NKI/Rockland sample versus data of 324 young healthy adults (164 females; mean age: $28.22 \pm 3.88$ ) from the Human Connectome Project (HCP) sample. 


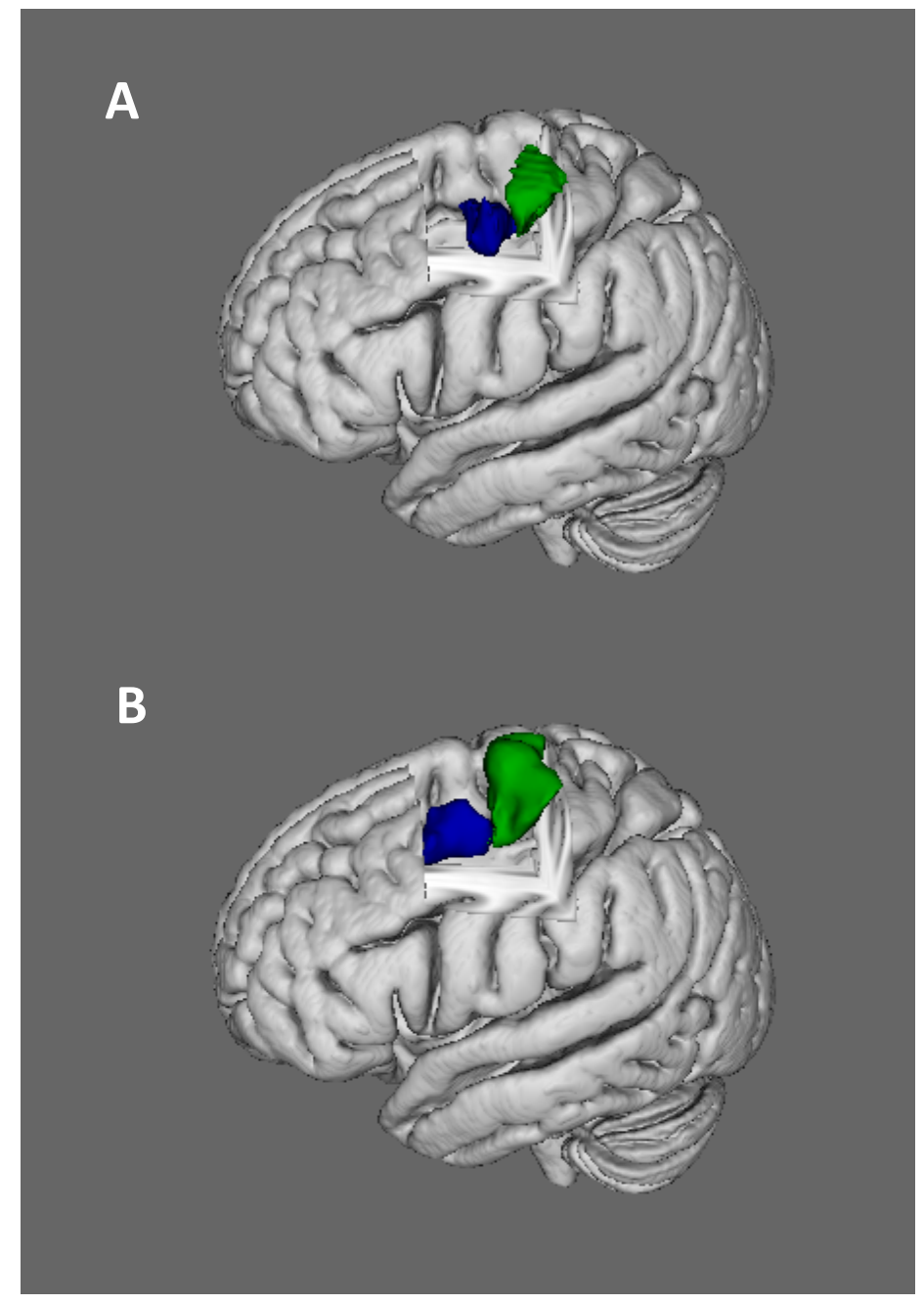

Figure S7. Illustration of the central and caudal cluster located in superior part of Area 6 (A) identified by multimodal CBP and the corresponding deep anterior $(6 \mathrm{~d} 3)$ and caudal $(6 \mathrm{~d} 1)$ subregions in superior part of Area 6 defined by cytoarchitectonic mapping (B, (Sigl et al. 2016)). 


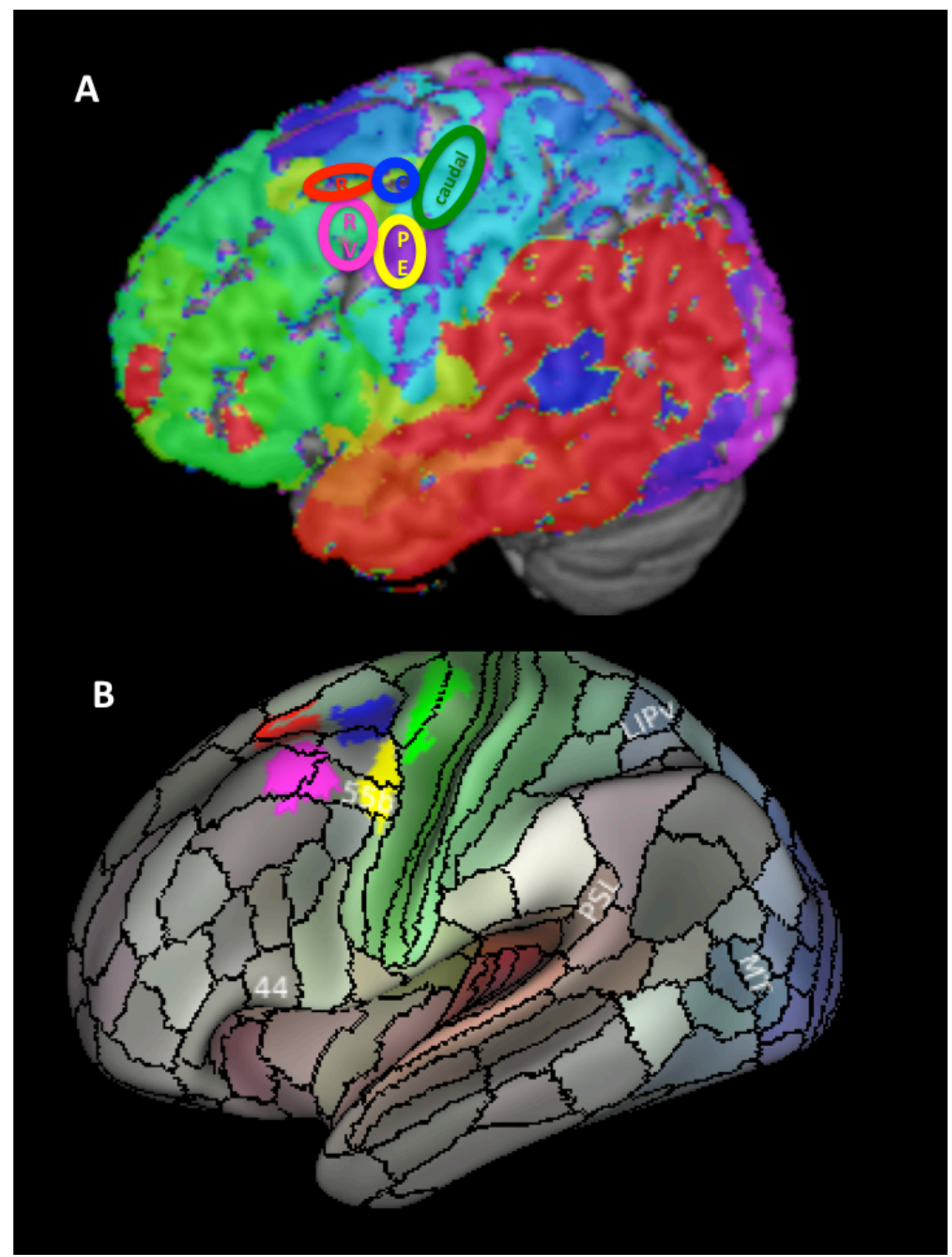

Figure S8. Overlay of the left PMd modules on Glasser et al. (2016)'s parcellation of the cerebral cortex. A. The surface map from Glasser et al. has been transposed to volume, the left PMd modules as volumes has been overlaid and manually drawn: red (R), rostral; blue (C), central; yellow (PE), ventral; pink (RC); rostroventral. Of note, the purple inferior PMd subregion overlapping with our assumed "left premotor eye-field (PE)" is the volume definition of Area 55b (cfr. Glasser et al.). B. The left PMd modules have been transposed to surface and overlaid on Glasser et al.'s surface with the Connectome Workbench: red, rostral; blue, central; green, caudal, yellow, ventral; pink; rostroventral. 


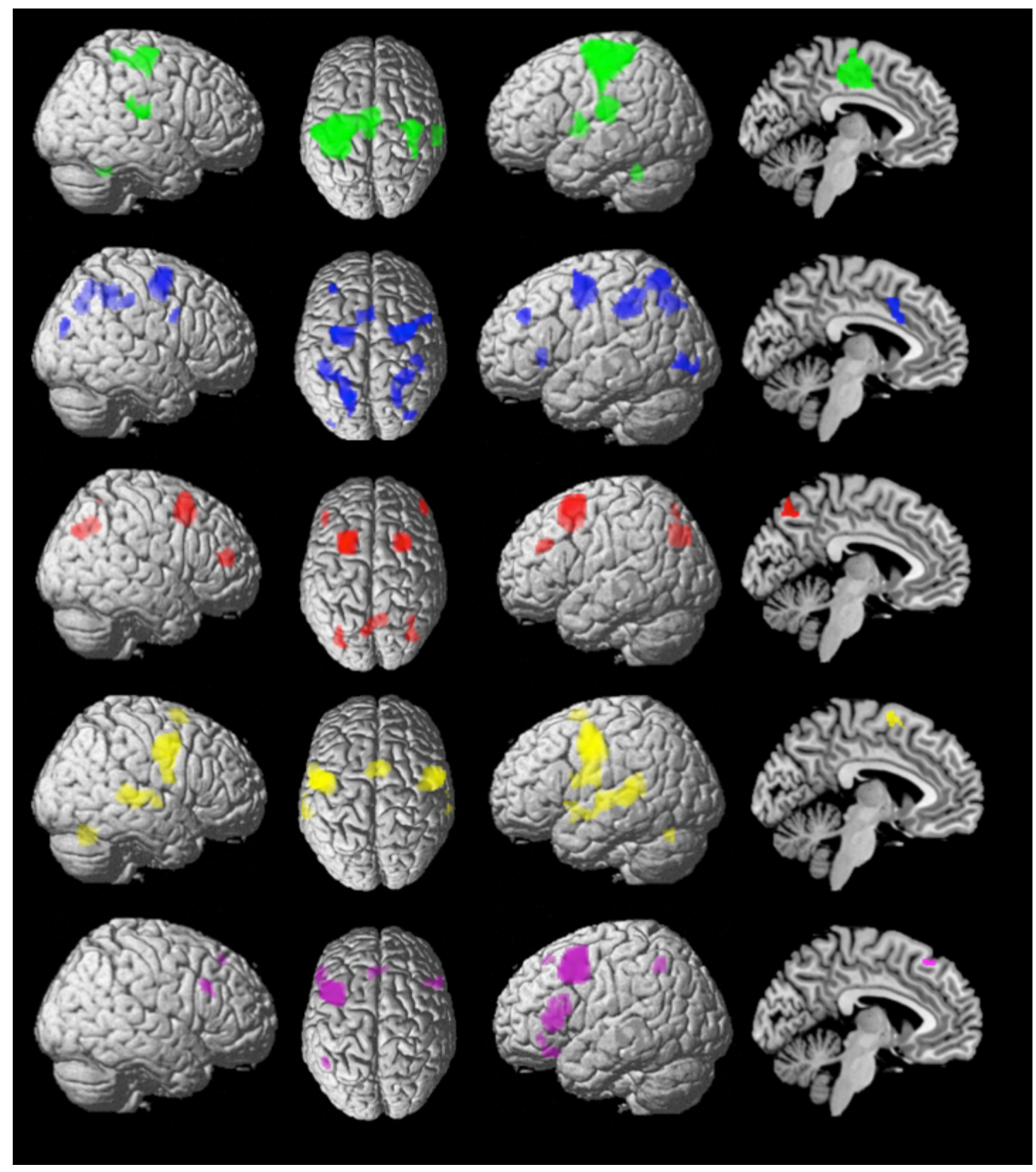

Figure S9. Task-based functional connectivity profile of the five left PMd modules revealed by MACM: green, caudal; blue, central; red, rostral; yellow, ventral; violet, rostroventral. 


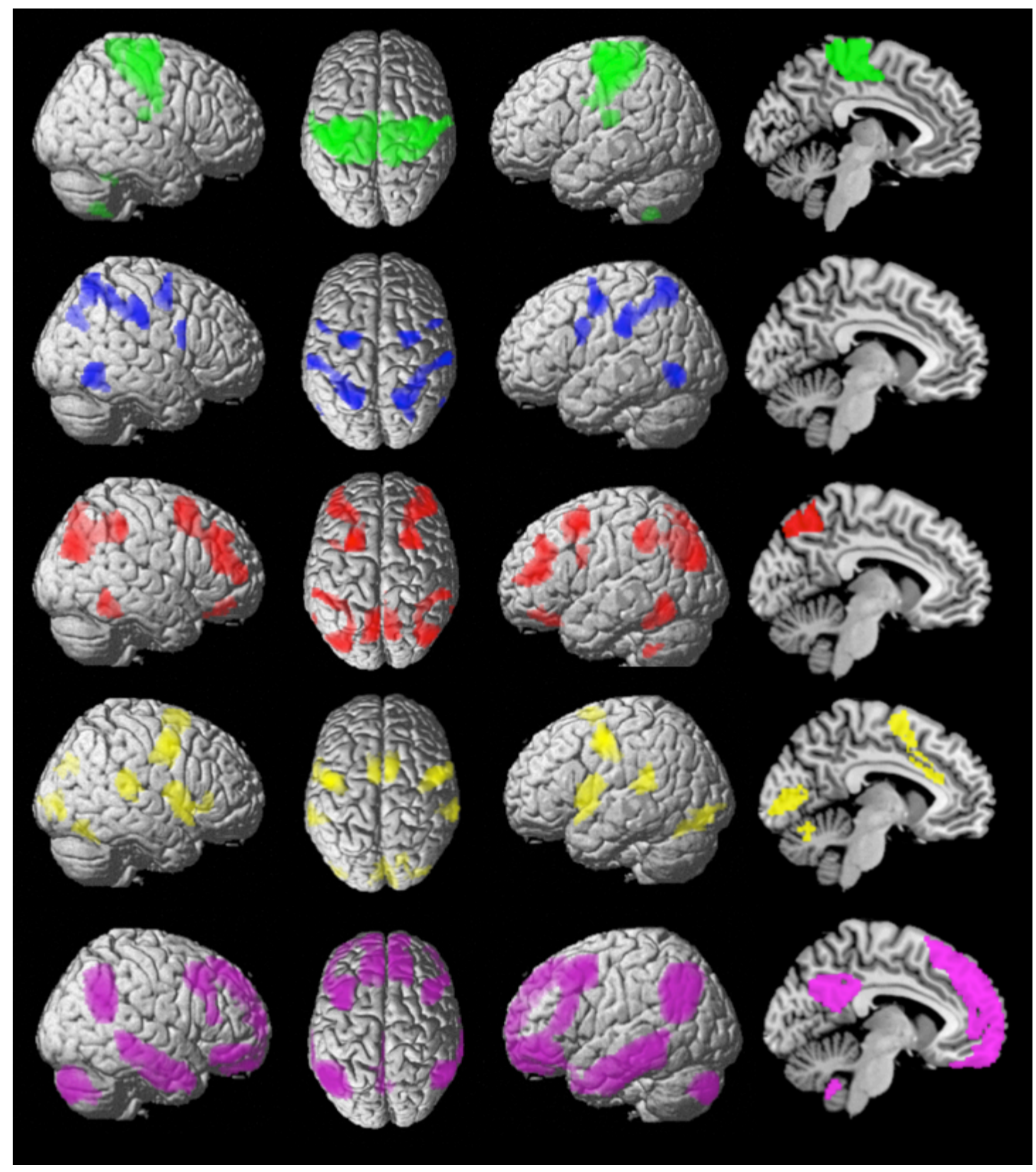

Figure S10. Unconstrained functional connectivity profile of the five left PMd modules revealed by RSFC: green, caudal; blue, central; red, rostral; yellow, ventral; violet, rostroventral. 
III.2. Five-cluster solution and six-cluster solution in the right PMd

CBP of the right PMd was performed with the improved methods (i.e. the same methods and dataset as used for the left PMd). First, our findings mainly replicate the results previously found in the right PMd with different pipelines. The 5-cluster parcellation of the right PMd with MACM-CBP and PDT-CBP replicated the pattern obtained in Genon et al. (2016) as illustrated in Figure S11 below. However, RSFC-CBP revealed a slightly different pattern from those revealed by the two other modalities and the one reported in Genon et al. (2016). That is, RSFC-CBP revealed a parcellation pattern that was specific to both the modality (i.e. RSFC) and the method used. This raises the question of the factors influencing RSFC-CBP and supports the need for multimodal CBP approaches (Glasser et al., 2016). Importantly, none of the 5-cluster parcellations found in the right PMd (neither in Genon et al.'s original study nor with the improved method here) mirrored the 5-cluster parcellation revealed in the left PMd. In particular, whereas the ventral part of the left PMd was already subdivided into two subregions with a substantial rostro-ventral subregion in the left PMd, this rostro-ventral subregion was not evidenced in any of the right PMd parcellations.

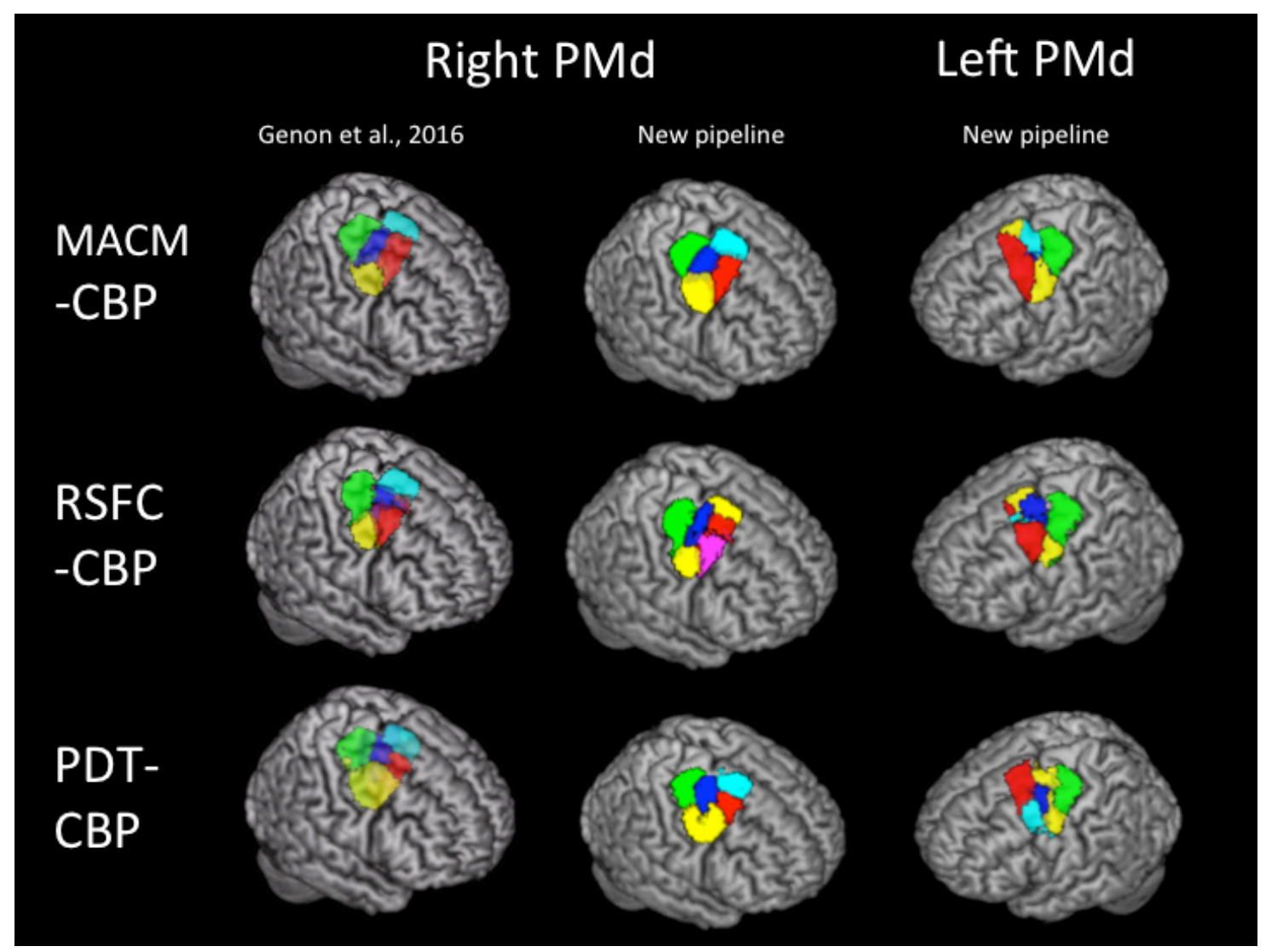

Figure S11. Multimodal CBPs of the right and left PMd with $\mathrm{k}=5$. 
Second, we further subdivided the right PMd into 6 clusters to investigate whether a rostroventral subregion could be consistently identified in the right PMd. This parcellation revealed that continuing to split the right PMd beyond stable subdivisions into five clusters could result in subdividing the ventral part of the right PMd. However, as illustrated in figure S12 below. this subdivision was not consistent across modality in the right hemisphere, as opposed to what we observed in the left hemisphere.

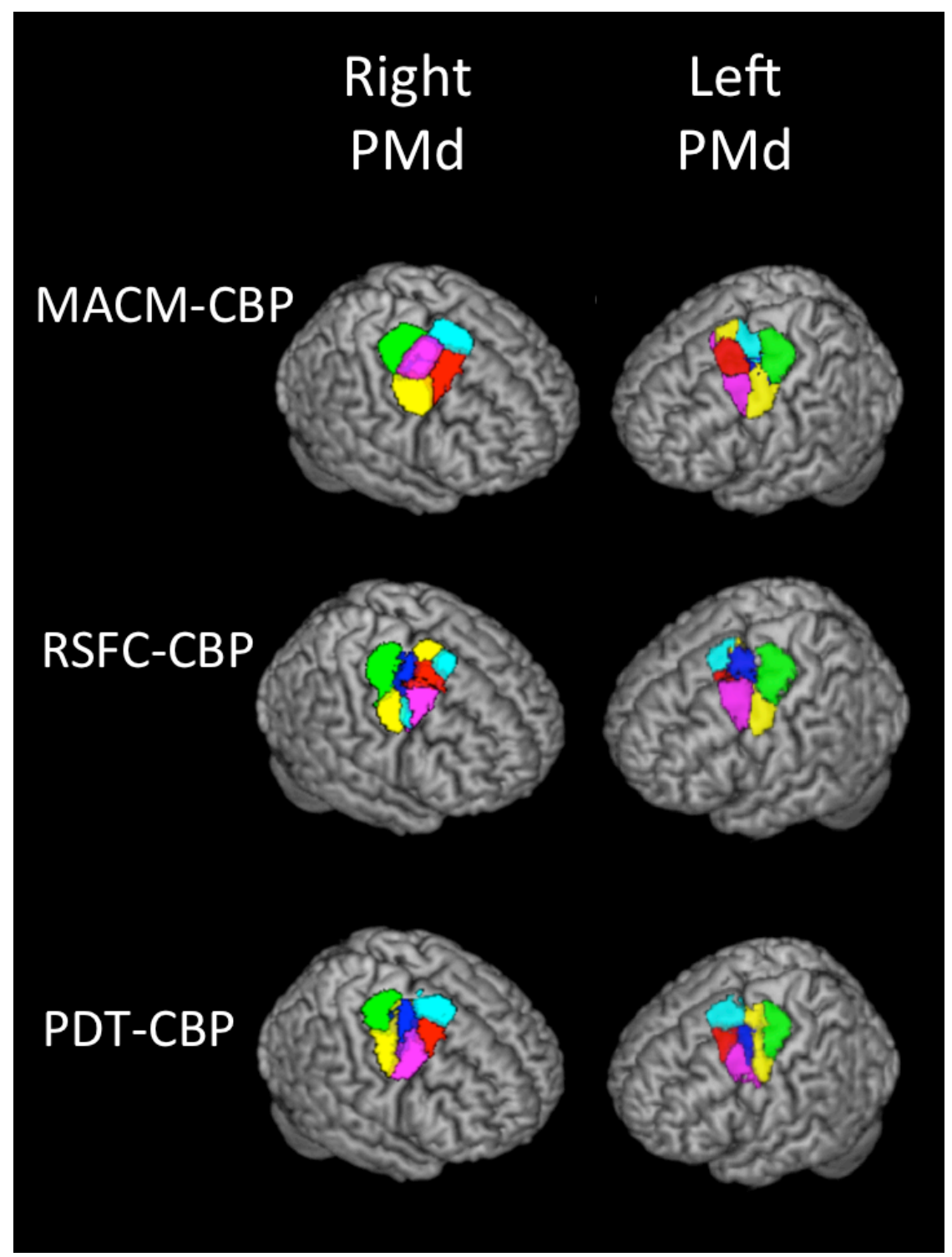

Figure S12. Multimodal CBPs of the right and left PMd with $\mathrm{k}=6$. 
III.3. Functional clustering across hemispheres

MACM-CBP was performed on a bilateral PMd VOI (figure S13). We examined a first subdivision $(\mathrm{k}=3)$, then stable coarse and stable higher subdivisions for the sake of completeness. Somewhat surprisingly, the first subdivision into three subregions (rostral, central, caudal) did not show a perfect symmetry across hemisphere with the rostral (assumed more "cognitive") part being larger in the left PMd when compared to the homotopic region in the right hemisphere. Then, already at coarse subdivision $(k=5)$, the rostral part of the left $\mathrm{PMd}$ (violet) including the rostro-ventral subregion was isolated as a distinct subregion from the PMd mosaic while the caudal (green), central (blue) and other rostral (red) subregion were shared by both hemispheres. Higher but stable subdivisions into 7 clusters further isolated the dorsal part of the right PMd as a distinct functional subregion in the PMd mosaic. In contrast, the ventral part of the right PMd remained as an uniform functional subregion lying from the anterior border to the posterior border and functionally similar to the ventro-posterior part of the left PMd but still clearly functionally different from the rostro-ventral left PMd. Finally, subsequent subdivision $(\mathrm{k}=8)$, split the atypic rostral left PMd into a rostro-ventral subregion and a rostral cluster. Of note, the pattern of functional similarities in the left and right PMd across scales suggest that there is a caudal-rostral gradient but asymmetric, with the most rostral parts being the most dissimilar in their functional connectivity profile across hemispheres.

For the sake of comprehensiveness, RSFC-CBP was also performed on the bilateral PMd VOI. Importantly, in line with the profile revealed by MACM at k = 3, RSFC-based first subdivision revealed a rostro-caudal, but asymmetric pattern, with the rostral (assumed more "cognitive") part being larger in the left PMd when compared to the right hemisphere. Importantly, only this very coarse subdivision across hemispheres was pointed out as the most stable clustering for several criteria. Nevertheless, we also examined higher subdivisions for the sake of completeness. Dividing the PMd voxels across hemispheres into five subregions suggested that the inferior part of the right and left PMd are not functionally similar in their intrinsic functional connectivity. Further subdividing the PMd voxels across hemisphere (into 7 and 8 clusters) finally clustered, respectively, the rostro-ventral subregions and the ventral subregions across hemispheres. However, this further splitting also resulted in a heterogenous (not spatially uniform) pattern of voxels assignments in the inferior part of the left PMd with several groups of deviant voxels. This observation again raises the question of the factors influencing RSFC-CBP. 


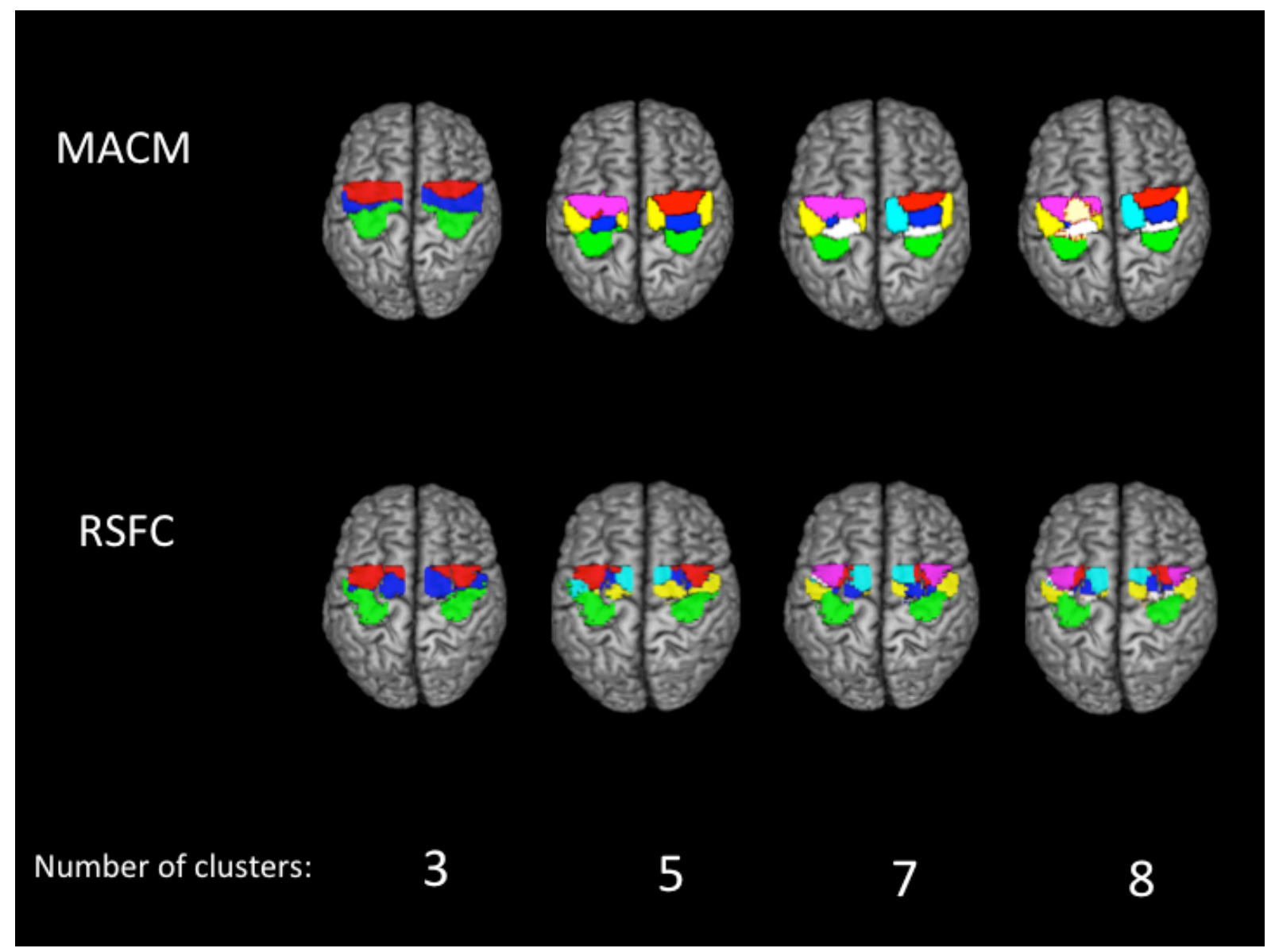

Figure S13. Functional clustering of PMd voxels across hemisphere when examining task based connectivity (MACM-CBP) and connectivity at rest (RSFC-CBP). 


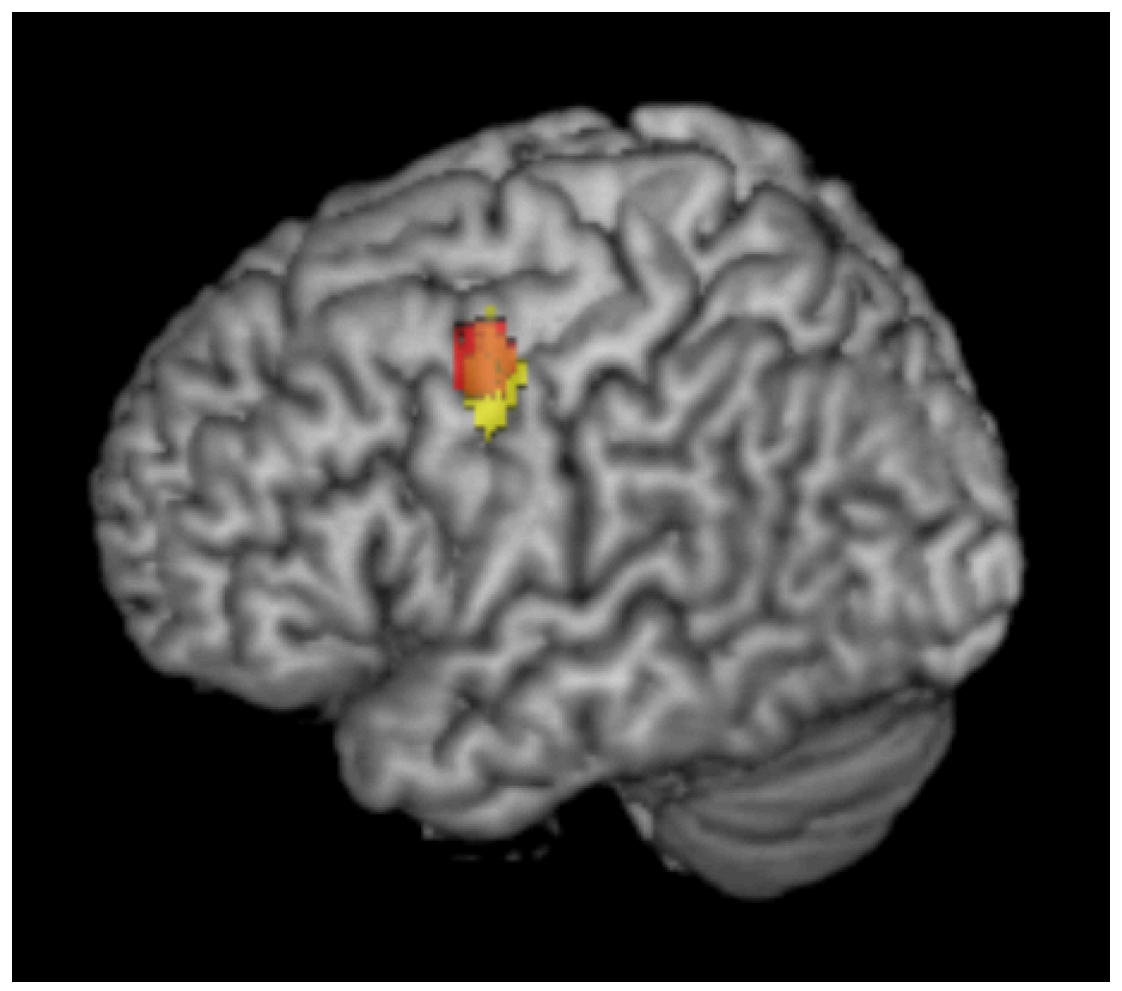

Figure S14. Rendering of the multimodal ventral left PMd module (yellow) and the cluster of activation for pro-saccades in Cieslik et al.'s (2016) meta-analysis (red). 

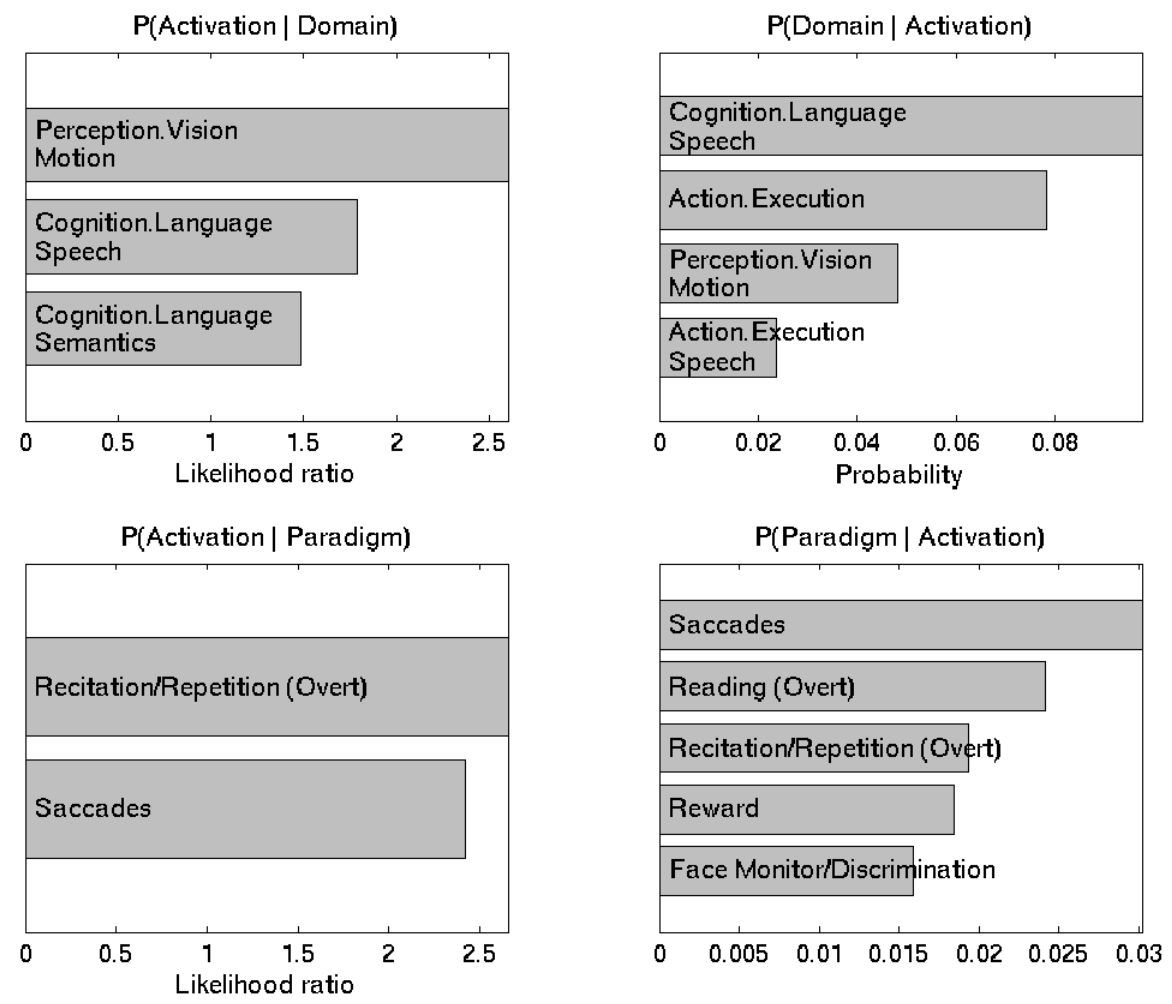

Figure S15. Functional behavioral characterization of Area 55b (volume definition) across the BrainMap database. Left side: forward inferences, Right side: reverse inferences, Upper histograms: Behavioral Domains, Lower histograms: Paradigm Classes. 

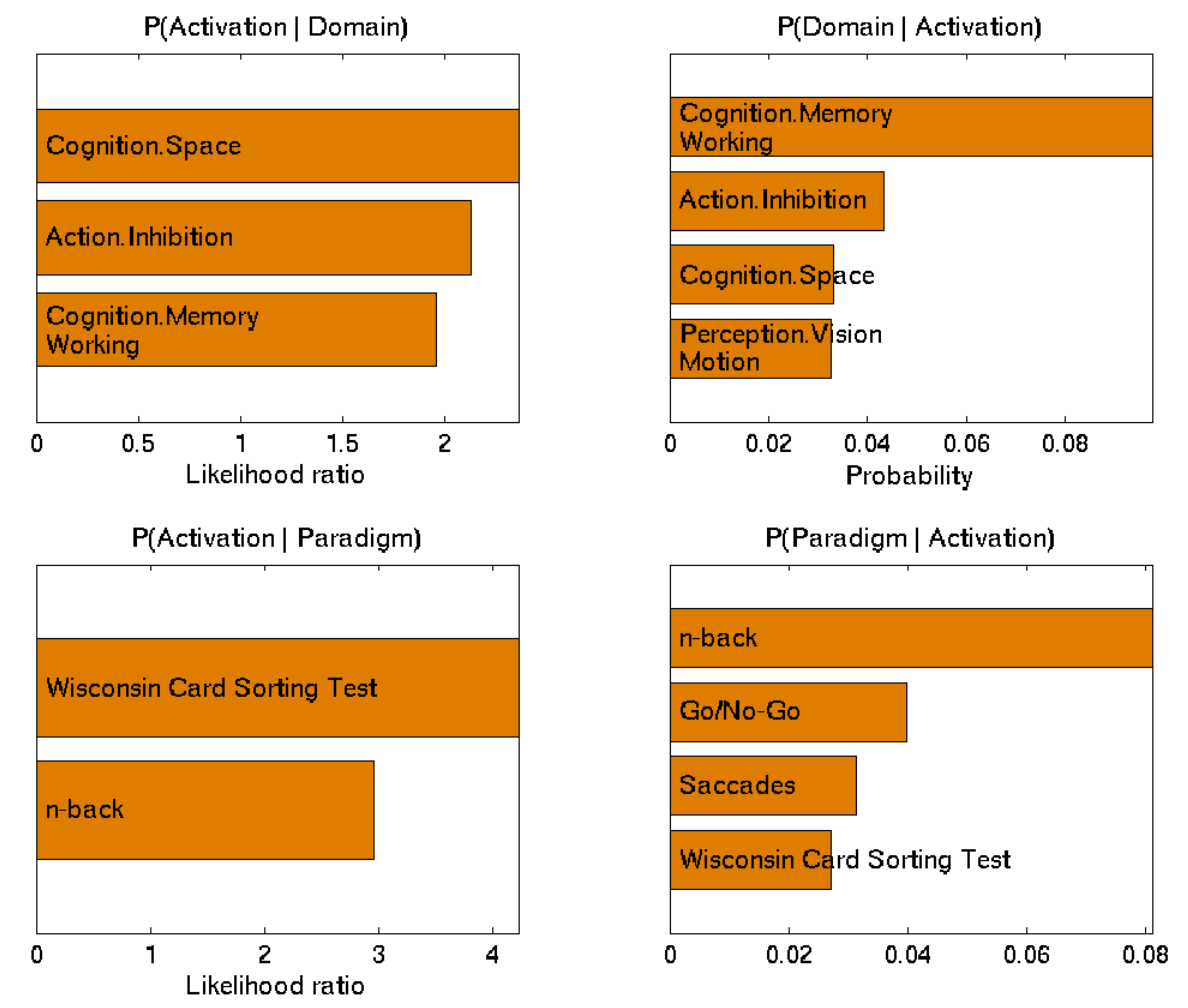

Figure S16. Functional behavioral characterization of rostro-ventral VOI in right hemisphere across the BrainMap database. Left side: forward inferences, Right side: reverse inferences, Upper histograms: Behavioral Domains, Lower histograms: Paradigm Classes. 
References:

Clos M, Amunts K, Laird AR, Fox PT, Eickhoff SB. 2013. Tackling the multifunctional nature of Broca's region meta-analytically: co-activation-based parcellation of area 44 . NeuroImage 83:174-188.

Genon S, Li H, Fan L, Muller VI, Cieslik EC, Hoffstaedter F, Reid AT, Langner R, Grefkes C, Fox PT, Moebus S, Caspers S, Amunts K, Jiang T, Eickhoff SB. 2016. The Right Dorsal Premotor Mosaic: Organization, Functions, and Connectivity. Cereb Cortex.

Glasser M, Coalson T, Robinson E, Hacker C, Harwell J, Yacoub E, Ugurbil K, Anderson J, Beckmann C, Jenkinson M. 2016. A Multi-modal parcellation of human cerebral cortex. Nature.

Sigl B, Caspers S, Mohlberg H, Cieslik EC, Eickhoff SB, Amunts K. 2016. The human dorsal premotor cortex - cytoarchitecture, maps and function. Proceedings of the OHBM 2016 Annual Meeting.

Tomassini V, Jbabdi S, Klein JC, Behrens TEJ, Pozzilli C, Matthews PM, Rushworth MFS, Johansen-Berg H. 2007. Diffusion-weighted imaging tractography-based parcellation of the human lateral premotor cortex identifies dorsal and ventral subregions with anatomical and functional specializations. The Journal of neuroscience 27:10259-10269.

Yeo BT, Krienen FM, Sepulcre J, Sabuncu MR, Lashkari D, Hollinshead M, Roffman JL, Smoller JW, Zollei L, Polimeni JR, Fischl B, Liu H, Buckner RL. 2011. The organization of the human cerebral cortex estimated by intrinsic functional connectivity. J Neurophysiol 106:1125-1165. 